\title{
I. Anfänge der Volksrichterausbildung
}

\section{Dokument 1}

Schreiben Bertz an das Sekretariat des Zentralkomitees der KPD; Berlin, den 14.12. 1945 SAPMO, ZPA, NL 182/1118, Bl.180, Original

\section{Werte Genossen!}

Anliegend einige Materialien, die den Beweis dafür erbringen, von wem die Initiative in verschiedenen, in der Deutschen Zentralen Justizverwaltung jetzt behandelten Fragen ausgegangen ist. Insbesondere ist es die Frage des Volksrichters, zu der Dr. Schiffer auch heute noch eine sehr unklare Stellung einnimmt und mit ihm ein Teil seiner „Volljuristen“. Ja, ich bin der Überzeugung, daß Dr. Schiffer den Volksrichter, wie wir ihn wollen, ablehnt. Das beweist unter anderem auch sein Artikel in "Der Morgen“ vom Freitag, den 14. Dezember ${ }^{1}$. Fest steht, daß Schiffer erst dann, als von Karlshorst ziemlich energisch eine Stellungnahme der Zentralen Justizverwaltung zur Frage des Volksrichters angefordert wurde, entsprechende Maßnahmen traf. Ihr seht also, was Dr. Schiffer mit seiner sonderbaren Bemerkung über meine Tätigkeit bezweckt ${ }^{2}$. Doch darüber bekommt Ihr demnächst noch weiteres Material.

Auch mein Vermerk über den Fragebogen zeigt, wie Schiffer gerade meine Arbeit sabotiert. Über seine „Personalpolitik“ habt Ihr bereits ein umfangreiches Material erhalten ${ }^{3}$.

Gleichzeitig füge ich bei das von mir gesammelte Material über den „Persönlichen Adjutanten“ Schiffers, Dr. Glüse".

Anlagen: 1) Die Entwicklung der Frage des Volksrichters in der Justizverwaltung,

2) Vermerk über Fragebogen ${ }^{6}$,

3) Material über Dr. Glüse ${ }^{7}$.

Mit komm[unistischem] Gruß

Bertz

${ }^{1}$ Es handelt sich um den Artikel "Justizreform“, in: Der Morgen, 14.12. 1945. Darin forderte Schiffer u. a. „einen neuen Typ für den Berufsrichter" und „einen neuen Typ [.. .] für den ihm beigegebenen Volksrichter", der ,aus einem bloßen Zierstück“ zu einem "vollwertige[n] Mitwirkende[n] an der Rechtsfindung werden" müsse; es ging Schiffer also um einen Laienrichter in Gestalt eines Schöffen oder Geschworenen.

${ }^{2}$ Schiffer hatte in einem Schreiben an Wilhelm Pieck vom 3.12. 1945 heftige Kritik an Bertz geübt und um dessen Entlassung gebeten, SAPMO, ZPA, NL 182/1118, Bl. 172.

${ }^{3}$ Mit Schreiben von Bertz vom 5.11. 1945, SAPMO, ZPA, NL 182/1185, B1.7-17.

${ }^{4}$ Bei Erich Glüse handelte es sich um den persönlichen Referenten von Schiffer, der nachweislich von September 1945 bis 31.7. 1946 in der DJV eingestellt war.

${ }^{5}$ Siehe Dokument 2.

6 Vermerk von Bertz, 1.12. 1945, SAPMO, ZPA, NL 182/118, Bl.171. Es geht darin um einen von Bertz ausgearbeiteten Fragebogen, der von Juristen vor der Wiedereinstellung zu beantworten war. Bertz warf Schiffer darin indirekt eine Verschleppung des Vorgangs vor.

7 Betrifft: Glüse, o.D., ebenda, Bl.181-186. Darin wirft Bertz Glüse vor, daß er - im Gegensatz zu seiner eigenen Darstellung - nie KPD-Mitglied gewesen sei, daß er sich bei Schiffer eingeschmeichelt habe, daß er gegen die Kommunisten in der DJV intrigiere, daß er Verbindungen zu den Briten, Amerikanern und Franzosen unterhalte und sich an Schiebereien von Lebensmitteln und anderen Gütern beteiligt habe. 


\section{Dokument $2^{1}$}

Aufzeichnung „Die Entwicklung der Frage des Volksrichters in der Deutschen Zentralen Justizverwaltung “, o.D.

SAPMO, ZPA, NL 182/1118, Bl.140-144, Original

Die Frage, daß die Richterschaft durch geeignete Kräfte aus Laienkreisen ergänzt werden muß, wurde innerhalb der Deutschen Zentralen Justizverwaltung unmittelbar nach dem Amtsantritt von Herrn Bertz mit den verschiedenen Mitarbeitern erörtert - Dr. Kleikamp, Melsheimer, Benjamin, Corsing u.a.

Auf Veranlassung von Herrn Bertz hatte daraufhin Dr. Corsing seine Gedanken zu dieser Frage in einer Notiz: „Zur Frage der Ernennung von Volksrichtern“ niedergelegt (24.September 45, Anl.1). Diese Notiz wurde den interessierten Mitarbeitern vorgelegt, von denen Dr. Melsheimer und Frau Benjamin auch ihrerseits schriftliche Anmerkungen dazu machten (Anl.2u. 3). Die Notiz wurde dann Herrn Dr. Schiffer zugeleitet, der darauf verfügte, daß er zu gegebener Zeit darauf zurückkommen und dann Herrn Dr. Corsing zur Beratung über diesen Punkt hinzuziehen werde.

Etwa Mitte Oktober fertigte Frau Benjamin nach erneuter Diskussion mit Herrn Bertz als private Arbeit einen Ausbildungsplan für Volksrichter und übergab ihn Herrn Bertz ${ }^{2}$.

Am 30./31. Oktober erhielt Dr. Melsheimer Kenntnis von mehreren "Verordnungen betr. Gerichtsverfassung“, die von Herrn Dr. Schiffer - obne Zuziehung der Gesetzgebungsabteilung - ausgearbeitet und bereits am 29./30. Oktober nach Karlshorst gegeben waren. In der „1.Verordnung betr. Gerichtsverfassung“ wollte er die Frage der Richterschaft wie folgt regeln:

\section{$\$ 3$}

Berufsrichter kann nur werden, wer entweder durch zwei Prüfungen den Vollbesitz von Rechtskenntnissen nachgewiesen hat oder den Vollbesitz von Rechtskenntnissen durch den Besitz anderer Eigenschaften ersetzt, die ihn zum Richter besonders geeignet erscheinen lassen.

Amtsrichter können auch Personen werden, die durch Ablegung einer Prüfung ein Mindestmaß von Rechtskenntnissen nachgewiesen und durch praktische Bewährung ihre Eignung für das Tätigkeitsgebiet eines Amtsrichters dargetan haben.

\section{$\$ 4$}

Als Volksrichter soll nur berufen werden, wer sich durch lauteren Charakter, gute Auffassungsgabe und sittliche Lebensführung Ansehen und Vertrauen bei seinen Mitbürgern erworben hat.

II.

Volksgerichtsbarkeit im Gebiet der Sowjetischen Besatzungszone in Deutschland.

1 Auszugsweise in: Amos, Justizverwaltung, S. 268-271.

2 Plan über die Ausbildung des Volksrichters, o.D., o.V., SAPMO, ZPA, NL 182/1185, Bl.29-37. Bertz sandte diesen Plan am 5.11. 1945 an Ulbricht, ebenda, Bl.6. 


\section{$\$ 6$}

Die staatliche Gerichtsbarkeit wird durch freigewählte Schieds- und Ehrengerichtsbarkeit ergänzt, über die eine besondere Verordnung ergehen wird.

Der Sinn dieses Entwurfs war folgender: es soll drei Gruppen von Richtern geben: Berufsrichter - Amtsrichter - Volksrichter. Bei den Personen, die auch ohne die vorgeschriebenen Prüfungen Berufsrichter werden können, sind nicht die Volksrichter in unserem Sinne gemeint, sondern es ist dabei an Verwaltungsbeamte, Bürgermeister und sonstige Akademiker gedacht - also Ersatz der Reaktionäre der einen Fakultät durch die der anderen. Die "Amtsrichter“ stellen die sogen. Rechtspfleger dar - mittlere Beamte, die mit der Wahrnehmung richterlicher Geschäfte beauftragt werden, und die sich besonderer Vorliebe Dr. Schiffers erfreuen. Die Volksrichter im eigentlichen Sinne will er dagegen auf die Aufgaben des Abschnitts II - die „Schieds- und Ehrengerichtsbarkeit“, beschränken.

Diese Gedanken entsprechen allerdings nicht ganz dem, was Dr. Schiffer in seinem Artikel im Morgen (Nr.53, 3. Okt. 45) dahin formulierte, daß jetzt die Stunde gekommen sei, in der es sich um ein Vorwärtsschreiten auf neuen Wegen zu neuen Zielen handelt ${ }^{3}$.

Nachdem Herr Bertz, Dr. Kleikamp und Dr. Melsheimer gegen dieses eigenmächtige Vorgehen Dr. Schiffers protestiert hatten, erhielt Dr. Melsheimer am 3. November von Dr. Schiffer den Auftrag, Denkschriften auszuarbeiten zu der Frage, wie der Umfang der Geschäfte der Gerichte dem verfügbaren Personal anzupassen sei - einmal durch Einschränkung in der Tätigkeit der Gerichte, andererseits durch Einbau der Volksrichter.

Die über diese Frage auszuarbeitende Denkschrift wurde auf Anregung von Dr. Melsheimer Frau Benjamin übertragen, die auf Anfordern von Herrn Bertz von der Abt. II, Herrn Dr. Winkelmann, für diese Arbeit ausdrücklich frei gestellt wurde. Diese Denkschrift (Anlage 4) wurde von Frau Benjamin gefertigt und am 6. November der Gesetzgebungs-Abteilung, Dr. Melsheimer, übergeben. Sie wurde von dieser den interessierten Abteilungen im Hause vorgelegt. Es äußerte sich dazu nur Herr Dr. Wende - Leiter der Abteilung Ausbildung u. Nachwuchs (Anlage 5).

Seine Äußerung wendet sich gegen die Bezeichnung "Volksrichter“, gegen die besondere Prüfungskommission ohne Beteiligung der Prüfungsämter (!) und macht in der Form grundsätzlicher Zustimmung gegen die Vorschläge zur Ausbildung im einzelnen Einwendungen, ohne selbst positive Vorschläge zu machen.

Diese Denkschrift wurde dann zusammen mit den Denkschriften über die Zuständigkeit der Gerichte Dr. Schiffer vorgelegt: Diese wurden nach Karlshorst weitergegeben die Volksrichter-Denkschrift jedoch nicht.

Am 19. November wurden die Abteilungsleiter - und auch Frau Benjamin - zu Dr. Schiffer gerufen; Herr Bertz wurde nicht eingeladen. Durch Herrn Dr. Rosenthal-Pell$\mathrm{dram}^{5}$ wurde ein soeben eingelaufenes Schreiben aus Karlshorst bekanntgegeben, in dem die bisher von Dr. Schiffer gemachten Vorschläge zur Schaffung und Heranbildung eines

${ }^{3}$ Eugen Schiffer, Rechtssicherheit in der Demokratie, in: Der Morgen, 3.10. 1945. Der Satz, auf den Bertz anspielt, lautet: ${ }_{n}$ Jetzt ist die Stunde gekommen, in der es sich nicht mehr um Umkehr und Rückkehr, sondern um ein Vorwärtsschreiten auf neuen Wegen zu neuen Zielen handelt."

4 Diese "Denkschrift über Möglichkeiten einer Einsparung von Richterkräften" wurde von Melsheimer angefertigt und am 9.11. 1945 zusammen mit der Denkschrift „Zur Frage des Volksrichters“ Schiffer vorgelegt: BAP, DP1 VA Nr. 2, Bl. 98-101.

${ }^{5}$ Erich Rosenthal-Pelldram (SPD), Leiter der Abt. I (Organisation der Gerichte). 
Richternachwuchses als ungenügend bezeichnet wurden und der Deutschen Zentralen Justizverwaltung aufgegeben wurde, innerhalb kürzester Frist einen Plan für die Errichtung von Schulen zur Heranbildung von Richtern in Lehrgängen in 6 Monaten auszuarbeiten; falls man dazu nicht in der Lage sei, solle man dies erklären ${ }^{6}$. Die Abteilung Ausbildung und Nachwuchs wurde mit der Ausarbeitung des Planes beauftragt, der dann am 20. November in demselben Kreise beraten wurde (vgl. auch Anl. 67).

Dieser Plan paßt sich einmal den russischen Forderungen in der Auswahl der Richter und der Organisation des Unterrichts an, in der Gestaltung des Stundenplanes weitgehend den Benjaminschen Vorschlägen. Er weicht davon ab vor allem in der Frage der Prüfungskommission (gegenstandslos durch das nach der russischen Forderung auf die antifaschistischen Parteien beschränkte Vorschlagsrecht), der praktischen Arbeit an den Gerichten und vor allem in dem Vorschlag, nach einer einleitenden allgemeinen Ausbildung die Ausbildung in eine solche für Strafrichter und Zivilrichter zu teilen. Von Dr. Melsheimer und Frau Benjamin wurden eine Reihe Abänderungsvorschläge im einzelnen dazu gemacht, denen z.T. stattgegeben wurde. Nicht zu erreichen war jedoch die Festlegung einer Ergänzungsausbildung für die bereits tätigen Laienrichter sowie die Nachschulung für die nach diesem Plan ausgebildeten Straf- oder Zivilrichter auf dem ihrer Ausbildung fehlenden Gebiet.

Dem Vernehmen nach soll dieser Plan jetzt Marschall Schukow vorliegen.

Einzelne Landesverwaltungen sind auf diesem Gebiet bereits vorgestoßen:

Das Land Sachsen geht nach einer Mitteilung der „Täglichen Rundschau“ vom 2. November (Anl. $7^{8}$ ) bereits daran, derartige Kurse einzurichten.

Die Provinz Sacbsen hatte nach einer Mitteilung ihrer Vertreter gelegentlich einer Rücksprache mit Herrn Bertz und Frau Benjamin bereits am 13. November eine Zahl solcher Volksrichter. Sie werden von den Berufsrichtern ihres Gerichts täglich 1-2 Stunden theoretisch unterwiesen, auch ist die bezirksweise Zusammenfassung der Volksrichter in Abständen von etwa 4 Wochen zu Kursen von achttägiger Dauer vorgesehen. Der Vizepräsident der Provinz Sachsen, Sievert [sic]', hat in einem Schreiben ausdrücklich erklärt, daß die Ausbildung systematisch erfolgen muß und er persönlich die Durchführung überwachen wird. Dies wird bestätigt und ergänzt durch die Meldung der "Deutschen Volkszeitung“ Nr. 156 vom 12.12., „Richter aus dem Volke - für Provinz Sachsen“ (Anl. ${ }^{10}$ ), wo es heißt: „Um dem Personalmangel abzuhelfen, aber vor allem auch, um die Justiz auf eine demokratische Basis zu stellen, sind in der Provinz Sachsen Richter aus dem Volke, die über genügend Fachkenntnisse verfügen, eingestellt worden. Die Ausbildung erfolgt durch antifaschistische Richter; die Leitung hat die Abteilung Justiz der Provinzialverwaltung der Provinz Sachsen."

6 Chef der SMAD-Rechtsabteilung an Chef der DJV, 14.11. 1945, geheim!, BAP, DP1 VA Nr.2, Bl.102-104. Zu dem Schreiben siehe Einleitung, S. 20.

7 Bei Anlage 6 handelt es sich um den Auszug eines Schreibens von Karassjow an Schiffer vom 27.10. 1945, in dem ein in diesem Zusammenhang irrelevantes Thema behandelt wird. Sie ist daher nicht abgedruckt. Der nach der Diskussion vom 20.11. 1945 geänderte Schulungsplan ist als Dokument 8 abgedruckt.

8 Auf den Abdruck dieser Anlage wurde verzichtet, da die Zeitungsnotiz auch in Dokument 6 zitiert wird.

9 Robert Siewert (KPD/SED), 1. Vizepräsident der Provinzialverwaltung Sachsen, dem die Personalabteilung und die Abteilungen Justiz und Polizei unterstanden.

${ }^{10}$ Auf den Abdruck wurde aufgrund des ausführlichen Zitats verzichtet. 
Für Mecklenburg, wo auch bereits eine Anzahl Laien eingesetzt sind, zeigte die Personalreferentin Frau Löhr ${ }^{11}$ bei einer Rücksprache mit Frau Benjamin am 26. Oktober großes Interesse für die Frage der Heranziehung weiterer Kreise zu Richtern und gab selbst wertvolle Anregungen.

Welcher Bedarf an Richtern (und Staatsanwälten) tatsächlich besteht, um allein die Gerichte ordnungsgemäß zu besetzen, mögen folgende Zahlen belegen:

Auf eine Anfrage nach dem gegenwärtigen Stand der Besetzung und dem Bedarf an die einzelnen Landes- bezw. Provinzialverwaltungen haben bis jetzt nur Brandenburg und Mecklenburg geantwortet. Die Angaben sind aber so gleichartig, daß sie einen Schluß auch auf die anderen Gebiete zulassen, zumal sie dem allgemeinen Bild entsprechen.

Mecklenburg verlangt sofort noch 76 Richter, Brandenburg 73. Sachsen will in seinen ersten Volksrichter-Lehrgang 80-100 Bewerber nehmen. Nimmt man als Durchschnitt 75-100, so ergeben sich für alle 5 Gebiete etwa 500 . Dies ist aber eine rein ziffernmäßige Feststellung und berücksichtigt nicht, daß

1) die gegenwärtige Besetzung nur eine Scheinbesetzung ist; die zur Zeit tätigen Richter sind überall zu etwa $60 \%$ über 60 Jahre alt, z.T. bis zu 75 Jahren. Von diesen wird daher in aller Kürze ein großer Teil wieder ausfallen,

2) die Richter zwar „Nicht-Pg's“ sind - aber damit noch keine zuverlässigen Antifaschisten. Darauf weist die Meldung aus der Provinz Sachsen, DVZ v. 12.Dezember ${ }^{12}$, besonders eindringlich hin.

Bei der Personalabteilung der Deutschen Zentralen Justizverwaltung sind an Meldungen von Juristen eingegangen im ganzen: 243. Davon sind zur Verwendung vorgemerkt als geeignet: 83(!), für die im übrigen noch die obengenannten Mängel der Überalterung und der politischen Fraglichkeit gelten.

Die Meldung der „Täglichen Rundschau“ aus Sachsen wurde erst nach etwa 8-10 Tagen in die Berliner Presse übernommen. Diese ohne Kommentar wiedergegebene Meldung veranlaßte dann den „Tagesspiegel“ vom 17. Nov. zu einem Artikel „Richter aus dem Volke" (Anl. $\left.7^{13}\right)$, in dem in wütenden Ausfällen gegen die sächsischen Pläne und gegen den Volksrichter überhaupt Stellung genommen wurde. Er spricht von dem „Galoppjuristen“ - eine Bezeichnung, die von reaktionären Kreisen auch aus der nächsten Umgebung von Dr. Schiffer mit merkbarem Wohlgefallen für diese Diskussion aufgegriffen wurde - einem "jungen Mann, der eben seine Nase in das Rechtswesen gesteckt hat", und empfiehlt, sich vor allem an die pensionierten Richter zu halten, und im übrigen den Plan „möglichst rasch im Papierkorb verschwinden zu lassen“.

11 Friedel Löhr (SED), Personalreferentin in der Abt. Justiz der Landesverwaltung Mecklenburg-Vorpommern.

12 Darin hieß es: „Ein großer Teil der Justizbeamten und -angestellten gibt noch nicht die Gewähr für eine demokratisch-antifaschistische Justiz. Die Bereinigung ist noch nicht abgeschlossen. In keiner anderen Behörde ist dies so notwendig wie bei der Justiz, denn die Gerichte entscheiden über Leben und Freiheit, Ehre und Familie und das Vermögen einer Person."

13 Nicht abgedruckt. Der Verfasser des Artikels stand, wie Bertz richtig darlegt, der Kurzausbildung von Richtern sehr kritisch gegenüber; er befürchtete im Zusammenhang mit den Volksrichtern einen Rückfall in die Praktiken der NS-Justiz. 


\section{Dokument 3}

Anlage 1, Stellungnahme zur Volksrichterfrage von Corsing; Berlin, den 24.9. 1945 SAPMO, ZPA, NL 182/1118, Bl.145-146, Abschrift

Es liegt durchaus im Bereich der Möglichkeit, daß sich die deutsche Justiz in naher Zukunft in die Lage versetzt sieht, bei der Ernennung von Richtern (gegebenenfalls auch von Staatsanwälten und Rechtsanwälten) über den engen Kreis der sogenannten Volljuristen hinauszugehen. Auf der einen Seite werden nicht genügend Personen vorhanden sein, die das Referendar- und Assessorexamen abgelegt haben, insbesondere weil viele Mitglieder des Richterstandes sich durch den Beitritt zur Nationalsozialistischen Partei kompromittiert haben, andererseits ist nicht zu verkennen, daß die gegen die Berufsrichter häufig voreingenommene Volksstimmung gebieterisch eine Erweiterung des Richterstandes verlangt, insbesondere weil die schwer zu bestreitende politische Einseitigkeit der Strafrichter während des dritten Reiches, aber auch schon in der Weimarer Republik eine tiefe Abneigung weiter Kreise der Bevölkerung gegen den Berufsrichter erzeugt hat. Diese Mißstimmung geht so weit, daß in der Bevölkerung ohne Zweifel eine volkstümliche, wenn auch nicht besonders scharf umrissene Rechtsprechung noch so gut abgefaßten Urteilssprüchen aus dem Kreise der Volljuristen vorgezogen wird. Der neue Staat kann aber nicht davon abgehen, eine Rechtsprechung zu schaffen, die auch den Zusammenhalt mit der Volksseele wahrt, muß vielmehr bestrebt sein, ein Gerichtswesen durchzusetzen, bei dem sich juristisches Können mit den geistigen und seelischen Strömungen des Volkes vereint.

Fragt man sich nun, wie es zu ermöglichen ist, den Kreis der Richter volkstümlicher zu gestalten, ohne zugleich die Qualität des Richterstandes zu senken, so ergibt sich, daß innerhalb der Bevölkerung außerhalb des bisherigen Volljuristenstandes sehr wohl ein Reservoir vorhanden ist, aus dem die Kräfte geschöpft werden können für eine zeitgemäßere Rechtsprechung. Es ist möglich, etwa aus folgenden Personenkreisen neue Richter und gegebenenfalls auch Staats- und Rechtsanwälte zu entnehmen:

a) In erster Linie sollte daran gedacht werden, den Kreis der sogen. Schmalspurjuristen heranzuziehen, d.h. solche Personen, die zwar nicht die beiden juristischen Prüfungen abgelegt und die praktische juristische Vorbereitungszeit vollständig absolviert haben, wohl aber doch durch ein gewisses Rechtsstudium juristische Kenntnisse erlangt haben, sei es, daß sie das Referendarexamen abgelegt und vielleicht einen Teil der praktischen juristischen Ausbildung zurückgelegt haben, sei es, daß sie das juristische oder nationalökonomische Doktorexamen bestanden haben. Dieser Personenkreis dürfte sich um so leichter dem Richterberufe anpassen, als er häufig aus Männern und Frauen besteht, die in ihrer praktischen Berufsarbeit etwa als Syndikus oder juristischer Beirat sich mit rechtlichen Materien jahrelang befaßt haben.

b) Auch Steuerberater, Wirtschaftsprüfer, Gewerkschafts- und Verbandssekretäre haben vielfach eine so umfassende Berührung mit juristischen Angelegenheiten, daß bei richtiger Auswahl aus ihnen ein gutes Material für den Justizdienst geschaffen werden könnte.

c) Das gleiche gilt für Bürobeamte etwa der Justizverwaltung, die sich in ihrem Dienste insbesondere etwa als Rechtspfleger oder in ähnlicher Position Erfahrungen auf dem Rechtsgebiet erworben haben; auch bei Kreissekretären und anderen mittleren Beam- 
ten von Verwaltungsbehörden wird vielfach eine höhere Lebenserfahrung vorhanden sein, die sie zum Amte eines Richters oder Staatsanwaltes befähigt.

d) Ähnlich liegt es bei Konkursverwaltern, Zwangsverwaltung, Rechtskonsulenten und Prozeßagenten sowie bei Bürovorstehern von Rechtsanwälten und Notaren, gegebenenfalls auch bei Gerichtsvollziehern.

e) Aber auch aus anderen Kreisen, wie z.B. bei Werkmeistern oder Polieren und in der erfahrenen älteren Arbeiterschaft dürften sich Menschen finden, die sowohl eine angeborene Begabung für Rechtsfragen [als auch] praktische Lebenserfahrung besitzen und diese schon als Schöffen, Geschworene oder als Mitglieder von Ausschüssen oder Berufsvereinigungen erworben haben.

Selbstverständlich müßte bei allen Kategorien geprüft werden, inwieweit die in Frage kommenden Personen die erforderlichen Voraussetzungen für die Stellung eines Richters, Staatsanwalts oder Rechtsanwalts erfüllen, d.h. ob sie ein solches Maß an allgemeiner Mittelbildung und Sprachgewandtheit besitzen, daß ihnen das öffentliche Auftreten in einer solchen Stellung zugemutet werden kann und ob ihre juristische Urteilskraft und Begabung ausreicht, die Aufgaben einer richterlichen Eingangsstellung voll zu erfüllen.

Die Feststellung dieser Voraussetzungen allein kann aber nicht genügen, um die so befähigten Personen ohne weitere Vorbereitung als Richter, Staatsanwälte oder Rechtsanwälte amtieren zu lassen. Wo selbst hochbegabte Rechtsstudenten nach mehrjährigem Studium zwei schwere Fachprüfungen ablegen, mehrere Jahre ernster, praktischer Vorbereitungsarbeit bei Gerichten, Staatsanwaltschaften und Rechtsanwälten leisten mußten, ohne selbst dann sofort fest angestellt zu werden, erscheint es bei der Einsetzung juristischer Außenseiter im Interesse der der Justiz unterliegenden Bevölkerung als eine Mindestforderung, die in Aussicht genommenen Außenseiter noch in möglichster Kürze für ihren neuen Beruf zu schulen, wenn man die Verantwortung für ein fruchtbares Funktionieren des so umgestalteten Gerichtsapparates übernehmen will. Es muß erreicht werden, daß sich diese nicht volljuristischen Volksrichter

1) eine wenigstens elementare theoretische Rechtskenntnis,

2) eine spezielle praktische Bewährung auf dem für sie vorgesehenen Rechtssektor erwerben.

Diese Rechtskenntnis und praktische Bewährung soll nicht irgendwie das Maß erreichen, das von den wissenschaftlich voll vorgebildeten Berufsrichtern verlangt wird, vielmehr wird es genügen, wenn den Anwärtern dasjenige Maß rechtlicher Kenntnisse und der Rechtstechnik vermittelt wird, das für die Anwendung der einfacheren Fälle des Zivilund Strafrechts unumgänglich erforderlich ist; neben diesem täglichen Brot der Rechtspflege müßten den Volksrichtern außerdem noch eine elementare Kenntnis des Zivil- und Strafprozesses vermittelt werden.

Die vorher erwähnte praktische Bewährung wird sich am bequemsten wahrscheinlich dadurch erreichen lassen, daß die Anwärter, bei denen die Voraussetzungen der Persönlichkeit und Befähigung festgestellt worden sind, etwa ein Jahr lang in Bürobeamtenstellungen der Gerichte und Staatsanwaltschaften zu arbeiten hätten und gleichzeitig von geeigneten Richtern, Staatsanwälten, Rechtsanwälten und gegebenenfalls auch Universitätsprofessoren in seminaristischen Kursen für den neuen Beruf geschult würden.

Bei einzelnen Kategorien, etwa zu a) bis c), könnte von Fall zu Fall ganz oder teilweise von der praktischen oder theoretischen Schulung abgesehen werden.

Die Gewinnung fruchtbarer juristischer Kräfte aus dem Volksganzen ohne volljuristische, wissenschaftliche Vorschulung würde sich danach vollziehen: 
1) durch Vorprüfung der Voraussetzungen für die Befähigung zum Richteramt überhaupt,

2) durch die praktische und theoretische Ausbildung und

3) gegebenenfalls durch eine Endprüfung, die den Anwärter für reif zur Einsetzung in ein Amt als Richter, Staatsanwalt oder Rechtsanwalt erklärt.

Dabei würde zu erwägen sein, ob das Ergebnis der Prüfung dahin zu ergänzen wäre, daß statt der Befähigung für das Richteramt usw. die einschränkende Berechtigung für eine Bürobeamtenstellung erteilt werden kann.

Berlin, den 24. September $1945^{1}$

\section{Dokument 4}

Anlage 2, Stellungnahme zur Volksrichterfrage von Melsheimer, o. D. SAPMO, ZPA, NL 182/1118, Bl.147, Abschrift

Den dankenswerten Ausführungen des Herrn Corsing ${ }^{1}$ trete ich durchaus bei. Ich fasse sie so auf, daß die gemachten Vorschläge dem Zwecke dienen, für die schwierige Übergangszeit, in der es an „Volljuristen“ fehlt, die nicht Pg. waren, durch Einstellung von „Volksrichtern“ Ersatz zu schaffen, daß aber an dem „Volljuristen“ als Richter grundsätzlich festgehalten werden soll. Den „volljuristischen“ Berufsrichter für die Zukunft so zu gestalten, daß er wahrhaft volksverbunden ist und volkstümlich Recht spricht, ist Aufgabe der Erziehung des juristischen Nachwuchses auf der Universität und im Vorbereitungsdienst; dazu wird noch viel zu sagen und noch mehr zu tun sein. Wird z. B. der junge Jurist nur ein zweijähriges Hochschulstudium und einen zweijährigen Vorbereitungsdienst durchzumachen haben, dann könnte er schon nach halbjährigem Vorbereitungsdienst als Hilfsrichter zur Verfügung stehen, also nach insgesamt 2 1/2-jähriger Ausbildung. Die auf Seite 1/2 der Denkschrift des Herrn Corsing unter a) erwähnten Referendare (möglicherweise sogar solche, die schon einen Teil des Vorbereitungsdienstes absolviert haben) sollten deshalb nicht zu „Volksrichtern“ gemacht werden; ihnen sollte - ev[entuell] unter Abkürzung des Vorbereitungsdienstes - die Gelegenheit gegeben werden, beschleunigt „Volljurist“ zu werden.

Alle anderen, die Herr Corsing erwähnt, sollten Volksrichter werden. Auch mit den Vorschlägen über Bildungsvoraussetzungen und Ausbildungsgang bin ich vorbehaltlich der Regelung im einzelnen einverstanden.

Dem "Volksrichter" sollten aber von vornherein die Aufgaben in der neuen Justiz zugewiesen werden, zu deren Lösung er nach seiner Vorbildung und nach der ihm zuteil gewordenen Ausbildung gut im Stande ist. Der „Volksrichter“, wie er mir vorschwebt, ist der in seinem Gerichtsbezirk geachtete und angesehene, als sachlich und gerecht bekannte Mann aus dem Volke. Ihm sollte deshalb bei den Amtsgerichten die Freiwillige Gerichtsbarkeit (besonders Vormundschafts- und Nachlaß-Sachen) sowie die sogenannte „Bagatellgerichtsbarkeit“ (Zivilsachen bis etwa 100,- Mk. oder 200,- Mk, Strafsachen bis etwa 1 Woche Gefängnis) übertragen werden. Höhere Objekte und wichtigere Sachen sollten

\footnotetext{
${ }^{1}$ Der Verfasser ist Fritz Corsing: siehe Dokument 2 und Einleitung, S. 16.

1 Dokument 3.
} 
an die Landgerichte gehen, wo der volljuristische Berufsrichter urteilt, wobei auch hier auf einen möglichst weitgehenden Einbau von Laienrichtern (Schöffen, Geschworenen) Bedacht genommen werden muß, und zwar nicht nur in der Strafgerichtsbarkeit, sondern auch in Zivilsachen, z.B. Ehesachen.

gez. Melsheimer

\section{Dokument $5^{1}$}

Anlage 3, Stellungnahme „Zur Frage der Volksrichter“ von Benjamin, o.D. SAPMO, ZPA, NL 182/1118, Bl.148, Abschrift

Mir scheint, daß eine begriffliche Klarheit geschaffen werden muß: Volksrichter ist der, der ohne jede spezielle juristische Vorbildung (auch der des mittleren Beamten) auf Grund seiner Lebenserfahrung, Berufs- und Menschenkenntnis in der Lage und besonders geeignet ist, Rechtsfälle selbständig zu entscheiden oder dabei mitzuwirken. (Dabei kommt ihm noch eine besondere Rolle in der Aburteilung faschistischer Verbrecher zu wie im Volksgericht des Landes Sachsen ${ }^{2}$.)

Nicht „Volksrichter" ist der, der, wie im Corsin'schen [sic] Vorschlag ${ }^{3}$, im Wege einer besonderen Ausbildung, die von der bisherigen abweicht, zum Richter vorgebildet wird. Diese Menschen sollten nach ihrer Vorbildung und der zusätzlichen Ausbildung als zum Richteramt befähigt im Sinne des $\$ 2 G^{2} G^{4}$ angesehen und entsprechend eingesetzt werden.

Notwendig ist im Augenblick beides:

Der Volksrichter, wie ihn auch Melsheimer ${ }^{5}$ kennzeichnet und sein Aufgabengebiet umschreibt, und der sofort eingesetzt werden muß (neben den Schöffen), und der beschleunigt und auf neuem Wege ausgebildete Richter.

Hierfür ist noch notwendig, daß ihre Existenzfrage während der Ausbildung geklärt wird, da es sich vorwiegend um Menschen handeln wird, die bereits im mittleren Alter stehen, eine Stellung gehabt haben oder noch haben, und die während der Ausbildungszeit womöglich mit einer Familie leben müssen.

$\mathrm{Zu}$ warnen ist nur bei beiden Kategorien vor juristischer Halbbildung.

gez. Benjamin

1 Das Dokument ist im Faksimile bereits wiedergegeben in: Im Namen des Volkes? Über die Justiz im Staat der SED. Katalog zur Ausstellung des Bundesministeriums der Justiz, Leipzig 1994, S. 32.

2 Am 2. Oktober hatte die Presse über ein Urteil des „Volksgerichts Dresden“ gegen NS-Verbrecher berichtet. Auch in Brandenburg existierten derartige Gerichte, die nicht in Befehl Nr.49 der SMAD zur Neuorganisation des Gerichtswesens aufgeführt waren: siehe Vermerk Kleikamps, 3.10. 1945, BAP, DP1 VA Nr. 2, Bl. 48.

3 Dokument 3.

4 \2 GVG lautete in der Fassung vom 22.3. 1924: „Die Fähigkeit zum Richteramte wird durch die Ablegung zweier Prüfungen erlangt. Der ersten Prüfung muß ein dreijähriges Studium der Rechtswissenschaft auf einer Universität vorangehen. Von dem dreijährigen Zeitraum sind mindestens drei Halbjahre dem Studium auf einer deutschen Universität zu widmen. Zwischen der ersten und der zweiten Prüfung muß ein Zeitraum von drei Jahren liegen, welcher im Dienste bei den Gerichten und bei den Rechtsanwälten zu verwenden ist, auch zum Teil bei der Staatsanwaltschaft verwendet werden kann." Reichsgesetzblatt 1924, S. 299.

s Siehe Dokument 4. 


\section{Dokument $\mathbf{6}^{1}$}

Anlage 4, Aufzeichnung „Zur Frage des Volksrichters“ von Benjamin, o.D. [gekürzt] SAPMO, ZPA, NL 182/1118, Bl.149-161, Abschrift

Unter Volksrichter (VR) ist jeder Richter zu verstehen, der, ohne den Voraussetzungen des $\$ 2 \mathrm{GVG}^{2} \mathrm{zu}$ entsprechen, das Amt eines Richters oder Staatsanwalts in vollem Umfange ausübt.

Durch die Entfernung der Pgs. aus den Gerichten sind Lücken entstanden, die aus dem Bestand an Juristen, die nicht Mitglied der NSDAP gewesen sind, allein schon ziffernmäßig nicht zu schließen sind. Eine Änderung des Grundsatzes, daß kein Pg. Richter sein darf (vgl. Befehl Nr. $49^{3}$ ), ist auch weder durch das Kontrollgesetz [sic] Nr. 4 vom 30. Oktober $1945^{4}$ noch die Entschließung der Blockparteien vom 3. November ${ }^{5}$ eingetreten.

Jedes Mitglied der NSDAP, das als Richter im Dritten Reich sein Amt unter der Kontrolle seiner Nazi-Oberen ausgeübt hat, ist ein Aktivist im Sinne dieser Bestimmungen mag auch für andere Zweige der Staatsverwaltung eine mildere Beurteilung Platz greifen.

Die Zahlen der Richter, die jedes Gebiet der Sowjetzone nach der endgültigen Entfernung der Nazis braucht, liegen im einzelnen genau nicht vor. Die folgenden Zahlen mögen jedoch ein gewisses Bild über die Lage geben:

Das Land Sachsen braucht zur ordnungsmäßigen Besetzung seiner Gerichte mindestens im ganzen 350 Richter; zur notdürftigsten Besetzung sind notwendig etwa 240, vorhanden sind etwa 170.

In der Provinz Sachsen mußten ausscheiden am 1.Oktober rund 100 Pgs., die ersetzt werden müssen.

Mecklenburg benötigt im ganzen 180 Richter - eine Zahl, die aber finanziell nicht getragen werden kann. Bereits tätig sind bis jetzt 40 , noch vorgesehen zunächst weitere 21 , diese Zahl ist jedoch keinesfalls ausreichend.

${ }^{1}$ Eine mit Marginalien Wendes versehene Kopie des Dokuments befindet sich in den Beständen des Bundesarchivs BAP, DP1 SE Nr.3478. Für den Wortlaut der Marginalien siehe Anm. 7-12.

2 Siehe Anm. 4 zu Dokument 5.

${ }^{3}$ Befehl Nr. 49 des Obersten Chefs der Sowjetischen Militäradministration vom 4.9. 1945, in: Um ein antifaschistisch-demokratisches Deutschland, S.142f. Absatz 3 legte fest: „Bei der Durchführung der Reorganisation des Gerichtssystems sind aus dem Apparat der Gerichte und der Staatsanwaltschaft sämtliche früheren Mitglieder der NSDAP zu entfernen, ebenso die Personen, welche unmittelbar teilgenommen haben an der Strafpolitik unter dem Hitler-Regime."

${ }^{4}$ Es handelt sich um Gesetz Nr. 4 des Alliierten Kontrollrats; es ist gedruckt in: Amtsblatt des Kontrollrats in Deutschland, Nr.2, 30.11. 1945, S.26f. Für diesen Zusammenhang siehe Art. IV, S. 27: "Zwecks Durchführung der Umgestaltung des deutschen Gerichtswesens müssen alle früheren Mitglieder der Nazipartei, die sich aktiv für deren Tätigkeit eingesetzt haben, und alle anderen Personen, die an den Strafmethoden des Hitlerregimes direkten Anteil hatten, ihres Amtes als Richter und Staatsanwälte enthoben werden und dürfen nicht zu solchen Ämtern zugelassen werden."

${ }^{5}$ Gemeint ist die Entschließung vom 30.10. 1945, die am 4.11. 1945 unter dem Titel „Strengste Bestrafung der Naziverbrecher" in der Deutschen Volkszeitung veröffentlicht wurde. Sie ist gedruckt in: Suckut, Blockpolitik, S. 92-94. Die einschlägige Passage lautet: „Die den aktivistischen Nazis persönlich aufzuerlegenden Maßnahmen und Leistungen sollen bestehen: a) im Ausschluß aus der öffentlichen Verwaltung und von der Betätigung in allen Stellungen, die ein öffentliches Vertrauen erfordern oder mit einer besonderen Verantwortung für das Wohl der Gesamtheit verbunden sind $[\ldots]$. 
Die Zahlen, die sich aus dem Bericht der Abt. II vom 27. Oktober ${ }^{6}$ ergeben, beweisen ebenfalls, daß die Zahl der Juristen, die nicht Pgs. waren, nicht ausreicht, um die Gerichte der ganzen Sowjetzone ausreichend und sachgemäß zu besetzen.

Nach diesem Bericht haben sich im ganzen an Bewerbern gemeldet: 176 Juristen, von denen 34 von vornherein abgelehnt wurden. Inzwischen hat der erste Ansturm an Bewerbungen beträchtlich nachgelassen, so daß mit einer entscheidenden Vergrößerung der Zahl der zur Verfügung stehenden Juristen nicht mehr zu rechnen ist.

Die Richter die nun jetzt bereits eingesetzt oder zum Einsatz vorgesehen sind, sind nun aber auch nicht ausnahmslos geeignet, und auch aus ihrer Zahl ist noch mit einem Abgang in nächster Zeit zu rechnen. Sie sind einmal überaltert. Schon aus rein physiologischen Gründen wird ein Teil von ihnen bald wieder ausscheiden.

Aus dem Bericht der Landesregierung Thüringen ergibt sich folgende altersmäßige Zusammensetzung für 73 der jetzt tätigen Richter, bei denen das Alter angegeben ist:

$\begin{array}{lrl}\text { unter 50 Jahren } & 34 & =47 \% \\ 50-60 \text { Jahre } & 19 & =26 \% \\ 60-65 \text { “ } & 12 & =16 \% \\ 65-70 \text { " } & 7 & =10 \% \\ \text { über 70 Jahre } & 1 & =1 \%\end{array}$

Aus der bei der Abt. II geführten Bewerberliste ergeben sich für 74 nicht abgelehnte Bewerber, soweit das Alter angegeben ist, folgende Zahlen:

$\begin{array}{lrl}\text { unter 50 Jahren } & 17 & =23 \% \\ 50-60 \text { Jahre } & 17 & =23 \% \\ 60-65 \text { " } & 19 & =26 \% \\ 65-70 \text { " } & 17 & =23 \% \\ \text { über 70 Jahre } & 4 & =5 \%\end{array}$

Bei diesen Zahlen handelt es sich um Durchschnittsergebnisse. Es ergibt sich daraus, daß schon der Bestand der jetzt eingesetzten Richter über $50 \%$ älter ist als 50 Jahre. Bei denen, die noch nicht eingesetzt sind und sich jetzt bewerben, sind über $75 \%$ über 50 Jahre und über $50 \%$ über 60 Jahre alt.

Diese Richter, die nicht der NSDAP angehört haben, stellen andererseits aber auch ideologisch zu einem Teil nicht das Richtertum dar, das der neue Staat braucht - denn allein die Tatsache, daß jemand nicht in der NSDAP war, macht ihn noch nicht zu einem Antifaschisten. Wenn wir auch noch kein allgemeines Bild über die Rechtsprechung in den einzelnen Ländern und Provinzen haben, so mögen die Berliner Verhältnisse herangezogen werden. In Berlin ist die Lage eher günstiger zu beurteilen als in der Provinz, da die Zahl der Richter, die nicht in der NSDAP waren, und wohl auch die Zahl derer, die gesinnungsmäßig wirklich Antifaschisten sind, hier [die] größere ist. Trotzdem wird der, der einmal den gegenwärtigen Gang der Berliner Gerichte beobachtet, und der andererseits so mit der Bevölkerung Fühlung hat, daß er ihre Stimme hört und Menschen aus allen Schichten zu ihm kommen, schon jetzt erkennen: die Gerichte haben noch nicht das Vertrauen des Volkes - oder sie sind in Gefahr, das Vertrauen, das ihnen in der ersten Zeit nach dem Umsturz spontan entgegengebracht wurde, schon wieder zu verlieren.

${ }^{6}$ Der Bericht konnte in den einschlägigen Akten nicht gefunden werden. 
Die Heranziehung von Volksrichtern ist also notwendig:

1) um in der zahlenmäßigen Besetzung die Gerichte arbeitsfähig zu machen,

2) um die Gerichte mit solchen Kräften zu besetzen, die wirklich das Vertrauen des Volkes haben.

Diese Heranziehung der Volksrichter, um die Gerichte überhaupt arbeitsfähig zu machen, ist um so notwendiger, als die Möglichkeit, den Geschäftsanfall bei den Gerichten durch Maßnahmen organisatorischer Art zu beschränken, durch das Gesetz Nr.4 des Allgemeinen [sic] Kontrollrates vom 30 . Oktober 45 ausgeschaltet, zumindest stark beschränkt ist. (Andererseits ist nicht anzunehmen, daß durch dieses Gesetz auch die Einführung des Volksrichters ausgeschlossen wird. Die in Art. I ausgesprochene Bezugnahme auf das GVG geschieht im Hinblick auf die „Umgestaltung der deutschen Gerichte“, d.h. auf den organisatorischen Aufbau, nicht aber die personelle Besetzung.)

\section{Wer soll Volksrichter werden?}

Das Bundesland Sachsen hat die Lösung der Frage des Volksrichters bereits praktisch in die Hand genommen. Die „Tägliche Rundschau“ Nr. 148 vom 2. November meldet:

„Auf Anregung der Sowjetischen Militärverwaltung für das Bundesland Sachsen soll wegen des Mangels an Richtern ein Lehrgang eingerichtet werden, in dem geeignete Kräfte aus allen Kreisen der Bevölkerung, Männer und Frauen, zu Richtern herangebildet werden sollen. Es kommen nur überzeugte Antifaschisten im Alter von etwa 25-35 Jahren in Frage, die nach ihren Fähigkeiten und Charaktereigenschaften die Gewähr dafür bieten, daß sie den Lehrgang mit Erfolg besuchen und späterhin ein Richteramt ausfüllen können."

Es ist richtig, daß die Kreise, die erfaßt werden sollen, so weit wie möglich gehalten werden. Nicht richtig scheint die Beschränkung der Altersklasse. Einmal ist ein Mindestalter von 25 Jahren zu niedrig: Dieser Altersklasse fehlt im heutigen Deutschland eigentlich jede Erfahrung außer der des Krieges. Der Volksrichter soll aber das, was er, zunächst wenigstens, weniger an juristischem Fachwissen hat, ersetzen durch allgemeine Erfahrung. Außerdem ist gerade diese Altersklasse die, die ideologisch den Einflüssen des Nazismus am stärksten ausgesetzt gewesen ist. In ihr werden „überzeugte Antifaschisten“ - eine unbedingt notwendige Voraussetzung - nur ausnahmsweise zu finden sein. Es scheint daher, wenn überhaupt eine Mindestgrenze festgesetzt und nicht jeweils von Fall zu Fall entschieden werden soll, das Mindestalter auf 30 Jahre heraufzusetzen zu sein. Da andererseits gerade die allgemeine Erfahrung und besondere Fachkenntnisse des Volksrichters nutzbar gemacht werden sollen, ist ein Höchstalter von 35 Jahren zu niedrig, und es wird vorgeschlagen, es auf 50 Jahre heraufzusetzen.

Ein besonderer Wert ist auf die Heranziebung der Frauen zu legen. Es ist zu berücksichtigen, daß Deutschland einen Frauenüberschuß von $70 \%$ hat, d.h. auf 100 Männer kommen 170 Frauen. Ein großer Teil der Rechtssuchenden sind daher auch Frauen, und dem muß auch in der Besetzung des Richteramtes Rechnung getragen werden. Dabei ist zu berücksichtigen, daß die Haltung des Nationalsozialismus den Juristinnen, vor allem den Richterinnen gegenüber besonders feindlich war, so daß der Nachwuchs an Berufsjuristinnen sehr gering ist, und die älteren Juristinnen in andere Berufe und die Anwaltschaft abgedrängt sind. Deshalb bietet gerade die Heranziehung als Volksrichter eine Möglichkeit, auch diesen Mangel in der Zusammensetzung der Richterschaft auszugleichen. 
Nach den vorliegenden Unterlagen sind bereits Volksrichter eingesetzt in:

Brandenburg 34 (anscheinend zum großen Teil noch von den Kommandanten)

Thüringen $\quad 4$ (mit einschlägiger Vorbildung wie Dr. jur., Direktor, Steuerberater)

Mecklenburg 12 (von 6 Staatsanwälten 5)

Prov. Sachsen 1

\section{Zulassung, Auswahl und Prüfung der VR}

Auf Grund der bei den Landesjustizverwaltungen oder der Zentralen Justizverwaltung vorliegenden Gesuche werden die Bewerber zur Zulassung von diesen Stellen einer besonderen Prüfungskommission vorgeschlagen. Auch die bereits tätigen Volksrichter werden von dieser Kommission nochmals auf ihre Eignung geprüft. Diese Kommission besteht aus $5 \mathrm{Köpfen:}$

2 Vertretern des öffentlichen Lebens auf Vorschlag der antifaschistischen Parteien, einem Vertreter der Gewerkschaften, einem Vertreter der Landesjustizverwaltung und einem Vertreter der Zentralen Justizverwaltung ${ }^{7}$. Die Kommission entscheidet auch darüber, ob ein Bewerber ganz oder teilweise von der Ausbildung freizustellen ist ${ }^{8}$. Sie nimmt auch die Abschlußprüfung ab, wobei die juristischen Mitglieder Angehörige der allgemeinen Prüfungskommission sind?.

Wie soll der Volksrichter eingesetzt werden?

Der VR soll zunächst nicht eingesetzt werden an einem kleinen Amtsgericht, wo er der einzige Richter ist. Es wird im übrigen individuell zu entscheiden sein: Beisitzer in Kammern des Landgerichts - besonders Frauen in Ehesachen - Beisitzer in Kammern für Handelssachen, in sonstigen Spezialkammern größerer Landgerichte. Amtsgerichte sollen möglichst so ausgewählt werden, daß noch mindestens ein Richter nach $\$ 2$ GVG da ist, der in Zweifelsfällen beraten kann. Als Richter der Freiwilligen Gerichtsbarkeit werden Beamte des gehobenen mittleren Dienstes sich besonders als Volksrichter eignen.

Um die augenblickliche Notlage zu beheben, die sofortige Abhilfe verlangt, kann mit dem Einsatz der Volksrichter nicht gewartet werden, bis sie ihre Ausbildung beendet haben, sondern ein Teil muß sofort eingesetzt werden und seine Ausbildung nebenher erhalten.

Wir haben daher für den Anfang 2 Gruppen von Volksrichtern zu unterscheiden:

1) die VR, die sofort zur Ausfüllung von Lücken, um den Mindestbedarf zu decken und den fürs erste notwendigen Betrieb aufrechtzuerhalten, herangezogen werden, und die keine vorberige Ausbildung erhalten, und

2) die VR, die eine vorherige Ausbildung erhalten und dann zur Befriedigung des weiteren Bedarfs sowie zum Ersatz sich als ungeeignet erweisender und auch sonst ausscheidender Richter herangezogen werden.

Diese zweite Form des VR wird jedenfalls so lange notwendig sein, bis wieder der normale Nachwuchs laufend zur Verfügung steht. $\mathrm{Ob}$ dann die Voraussetzungen für die Sonderausbildung des VR fortfallen, wird davon abhängen, inwieweit dann dieser Nachwuchs die Gewähr dafür bietet, daß er die Eigenschaften des Berufsrichters mit denen des VR vereint $^{10}$.

\footnotetext{
7 Marginal Wendes: „nach welchen Gesichtspunkten?"

${ }^{8}$ Marginal Wendes: „Sache der Justizverwaltung!“

"Marginal Wendes: „nur 2 Juristen!“

${ }^{10}$ Marginal Wendes: „Dann gibt es also eines Tages vielleicht nur noch Volksrichter."
} 
Allgemeines zur Ausbildung der Volksrichter

Als geeignete Ausbildungsstelle erscheint die jeweilige Landesuniversität in Verbindung mit dem in ihrem Ort liegenden Land- oder Oberlandesgericht. Dafür spricht: An den einzelnen Gerichtsorten - auch an den Landgerichten - sind unter den heutigen Verhältnissen kaum Richter anzutreffen, die einmal genügend Zeit haben und andererseits auch befähigt sind, die Ausbildung der VR zu leiten. Die juristischen Fakultäten werden auf Grund der neuen Studienpläne für das juristische Studium auch ideologisch genügend vorbereitet sein $^{11}$, um die Ausbildung der Volksrichter zu einem wesentlichen Teil zu tragen. Ergänzt muß die Tätigkeit der Fakultät werden durch die der Praktiker von den Gerichten, die in den Universitätsstädten von jeher mit den juristischen Fakultäten in Verbindung gestanden haben.

Diese Konzentration der Ausbildung der VR an den Universitäten bietet verhältnismäBig geringe Schwierigkeiten für die VR, die ihre Ausbildung im voraus erhalten. Sie können - es wird sich um Kurse von höchstens jeweils 30-40 handeln - in der Universitätsstadt zusammengezogen werden, und es wird auch ihre Verteilung zur praktischen Anleitung (vergleiche unten S.[10]) auf die dort vorhandenen Gerichte möglich sein.

Wesentlich schwieriger liegt die Frage der ohne vorherige Ausbildung tätigen VR. $\mathrm{Ob}$ eine wirksame Schulung für sie bereits jetzt durchgeführt werden kann, hängt in erster Linie von den Verkehrsverhältnissen ab. Es wird vorgeschlagen, diese VR in der Universitätsstadt zu Wochenendkursen (Sonnabend-Sonntag) zusammenzufassen.

Für Brandenburg müßte Potsdam gewählt werden. Dort werden die Kurse durch qualifizierte Mitglieder des Oberlandesgerichts und der Justizabteilung gehalten werden können, und Berliner Professoren können zur Abhaltung von Vorlesungen auch nach dort kommen. Das ist aber nur möglich, wenn sie in der Lage sind, durch Kraftwagen oder erträgliche Eisenbahnverbindung in etwa 2-3 Stunden die Universität zu erreichen. Diese Möglichkeit wird bestehen in den beiden Sachsen, vermutlich zum Teil in Thüringen, ist aber nach den vorliegenden Berichten zur Zeit noch fast undurchführbar für Teile von Brandenburg und Mecklenburg.

Es ist deshalb von mecklenburgischer Seite vorgeschlagen worden, etwa alle 2 Monate einen 10-Tage-Kursus für die VR abzuhalten. Es würde dies, so lange eine Besserung der Verbindungsmöglichkeiten nicht gegeben ist, ein Ausweg sein, der aber wegen der Beschränkung der Ausbildungsmöglichkeit (vergl. unten S.13) nur als vorübergehende Notlösung anzusehen ist.

Als Grundsatz für die Ausbildung der VR, die sofort eingesetzt sind, ist aufzustellen: dezentralisierter Einsatz - zentralisierte Ausbildung.

Als Grundgedanke für die Ausbildung der VR gilt: Die Richter kommen aus dem praktischen Leben. Es sind bei ihnen, auch wenn sie nicht als Kaufleute, Lehrer oder frühere Justizangestellte und ähnliches eine gewisse theoretische Vorbildung haben, sondern Gewerkschafter, Monteure, Werkmeister waren, allein schon aus ihrer Erfahrung gewisse Vorkenntnisse da. Was sie brauchen, ist einmal ein fester Bestand an positivem Wissen im materiellen und im Verfahrensrecht. Daneben müssen sie eine allgemeine rechtspolitische Schulung erhalten, die sie die gegenwärtige Form dieses Rechts erkennen läßt als Ausdruck der gegebenen wirtschaftlichen und gesellschaftlichen Verhältnisse. Dazu muß kommen eine Unterrichtung über die laufende neue Gesetzgebung und ihre Bedeutung und die aktuellen Fragen der Rechtsprechung.

11 Wende versah diesen Passus mit einem Fragezeichen. 


\section{Plan für die Ausbildung der Volksrichter}

\section{Vorherige Ausbildung}

Es wird zunächst eine Ausbildung von 6 Monaten vorgeschlagen. Die Ausbildung kann je nach den Vorkenntnissen des Anwärters eine gewisse Beweglichkeit haben und evtl. auch durch Universitätsvorlesungen nach Wahl ergänzt werden. Es wird folgende Gestaltung des Planes vorgeschlagen:

\begin{tabular}{|c|c|}
\hline Bürgerliches Recht & 8 Wochenstunden \\
\hline Zivilprozeß & $4 \quad m$ \\
\hline Strafrecht & 5 \\
\hline Strafprozeß & 3 \\
\hline Freiwillige Gerichtsbarkeit & 1 \\
\hline Strafvollzug & 1 \\
\hline $\begin{array}{l}\text { Verbrechensursachen und Verbrechensbekämpfung } \\
\text { Einführung in die Volkswirtschaft unter }\end{array}$ & 2 \\
\hline Berücksichtigung der Arbeiterbewegung & ” \\
\hline Recht und Rechtsprechung der Gegenwart & 2 \\
\hline
\end{tabular}

Im einzelnen ist dazu zu bemerken: Der ganze Unterricht ist weniger als Vorlesung, sondern als seminaristische Übung zu betreiben. Deshalb sind die Stunden, die sich mit dem positiven Recht befassen, nicht in Vorlesungen und Übungen aufgeteilt, dies ist vielmehr dem Lehrer überlassen. In den Prozeßübungen wird man nicht nur einen Teil theoretisch lösen, sondern auch Verhandlungsführung, Anklagevertretung usw. üben.

Die Stunde über Freiwillige Gerichtsbarkeit soll nur einen Überblick geben, da für den praktischen Einsatz in diesem Fach solche Bewerber verwandt werden sollen, die als mittlere Beamte aus der Justiz kommen und gerade dieses Gebiet praktisch beherrschen.

Die Vorlesung über Verbrechensursachen und -bekämpfung soll einmal in die sozialen Ursachen des Verbrechens, andererseits in die praktische Zusammenarbeit mit der Kriminalpolizei einführen.

Unter dem Titel „Einführung in die Volkswirtschaftslehre“ ist die soziologische Einführungsvorlesung zu verstehen.

In der Vorlesung „Recht und Rechtsprechung der Gegenwart" sollen insbesondere auch die Fragen behandelt werden, die jetzt praktisch vor dem Richter stehen: Behandlung der Pgs., Beschlagnahmen, Wohnungsräumungen und -einweisungen, sog. „Plünderungen“, "Amtsanmaßungen“" usw.

Neben dieser theoretischen Schulung hat der praktische Einsatz an den Gerichten des Ortes zu erfolgen ${ }^{12}$, und zwar täglich 3-4 Stunden. Sie wird in zwei Abschnitte von je 3 Monaten geteilt: Die ersten drei Monate wird die Tätigkeit eines mittleren Beamten umfassen. Kanzlei-, Registraturarbeiten, Protokoll führen, während in der zweiten Hälfte der Ausbildungszeit der Bewerber die Stellung eines Referendars haben soll, insbesondere also auch schon als Richter kraft Auftrags tätig werden kann.

${ }^{12}$ Marginal Wendes: „ohne weitere Schulung?“ 


\section{Gleichzeitige Ausbildung}

a) Wochenendkurse:

Hierbei wird zunächst davon ausgegangen, daß der Richter als erstes positives Wissen auf dem Gebiet gebraucht, auf dem er tätig ist: also entweder als Strafrichter oder als Zivilrichter. Aus dem Grunde wird es auch nicht zweckmäßig sein, einen solchen VR als alleinigen Richter, wo er alles gleichzeitig erledigen muß, anzustellen.

Es werden demnach Strafrichter und Zivilrichter getrennt ausgebildet, jeweils 6 Monate, und zwar zuerst auf ihrem unmittelbaren Arbeitsgebiet und dann auf dem anderen. Die ganze zusätzliche Ausbildung soll also 12 Monate umfassen, 6 Monate als Strafrichter und 6 Monate als Zivilrichter.

Sonnabend:

Plan für die Strafrichter

Übersicht über das bürgerliche Recht

mit Fällen

Strafrecht

Strafprozeß

Strafvollzug

Verbrechensursachen und -bekämpfung

\begin{tabular}{l}
1 Stunde \\
$3 "$ \\
$2 "$ \\
$1 \%$ \\
$1 \%$ \\
\hline 8 Stunden
\end{tabular}

Sonntags:

Einführung in die Volkswirtschaftslehre mit

Berücksichtigung der Arbeiterbewegung

2 Stunden

Recht und Rechtsprechung der Gegenwart

$\frac{2}{12 \text { Stunden }}$

Sonnabend:

Bürgerliches Recht

Übersicht über das Strafrecht

Zivilprozeß

Freiwillige Gerichtsbarkeit

Plan für die Zivilrichter

Sonntags:

Wie oben. Diese Vorlesungen können evtl. für beide Gruppen gemeinsam abgehalten werden.

b) zweimonatliche 10-Tage-Kurse:

Diese Kurse sind auch zu trennen für Straf- und Zivilrecht und so zu gestalten, daß sie jeweils einen abgeschlossenen Fragenkomplex behandeln. Auch in diesem Rahmen sind die allgemeinen Vorlesungen durchzuführen.

Der sechsmonatige Kursus umfaßt nach dem obigen Plan 728 Lehrstunden. Die Wochenendkurse enthalten in je 6 Monaten 312 Stunden. Der vollständige Lehrstoff würde also in 12 Monaten 624 Stunden umfassen, dem 6-Monats-Kursus also um rund 100 Unterrichtstunden nachstehen.

Die alle zwei Monate abzuhaltenden 10-Tage-Kurse würden aber im ganzen Jahre nur 60 Unterrichtstage umfassen; selbst wenn man auf jeden Tag 8 Stunden legte - für die Auf- 
nahmefähigkeit zu viel - ergäben sich im Jahre nur 480 Stunden, auf denen natürlich nur ein geringerer Teil des Lehrstoffes gebracht werden kann.

Diese Kurse müßten daher unterstützt werden durch eine Art Unterrichtsbriefe, die die Zentralverwaltung gemeinsam mit den Landesverwaltungen ausarbeitet und die zweiwöchentlich den VR zugehen.

$[\ldots]^{13}$

\section{Dokument 7}

Anlage 5, Schreiben Wende an Melsheimer; Berlin, den 12.11. 1945

SAPMO, ZPA, NL 182/1118, Bl.162-163, Abschrift

Von der Denkschrift zur Frage des Volksrichters ${ }^{1}$ habe ich mit um so größerem Interesse Kenntnis genommen, als für den wichtigsten Teil der in ihr behandelten Probleme, die Ausbildung der Volksrichter, offenbar Abt.VI zuständig ist. In Anbetracht dessen darf ich zu Ihren Ausführungen wohl auch sachlich Stellung nehmen, wenn auch zunächst nur vorläufig.

Den Entschluß, neben den „gelehrten“ Richtern eine zweite Kategorie Richter zu entwickeln, nehme ich als Ausgangspunkt ohne weitere Stellungnahme hierzu an, wenn ich auch empfehlen möchte, eine andere Bezeichnung für die neue Art Richter zu wählen. Ich fürchte, daß der sogenannte gelehrte Richter, der schließlich auch im Namen des Volkes Recht spricht, also auch ein Volksrichter ist, im Urteil weiter Bevölkerungskreise nun erst recht als nicht genügend volks- und lebensnahe angesehen und dadurch in einen sicher von niemand [sic] gewollten Gegensatz zu den „wahren“ Volksrichtern gestellt werden wird, eine Entwicklung, die von allen Seiten verhindert werden sollte.

Ich würde es auch sehr begrüßen, wenn man - wie ich Ihren Ausführungen Seite 6 unten, 7 entnehmen möchte - davon absähe, die Volksrichter von vornherein auf bestimmte Aufgabengebiete nur der ersten Instanz zu beschränken. Ist der ausgebildete Volksrichter überhaupt als Richter geeignet, so muß ihm meiner Meinung nach genau wie dem "gelehrten" Richter jedes Aufgabengebiet und in jeder Instanz offen stehen, wenn er sich entsprechend bewährt, evtl. also auch z. B. der Vorsitz in einer Kammer für Handelssachen, wenn er auch kraft seiner Erfahrung im kaufmännischen Beruf die nötigen Vorbedingungen dafür erfüllt. Daß besonders Frauen in Ehesachen geeignet sein sollten, kann ich auf Grund vieljähriger Praxis auf diesem Gebiet allerdings nicht anerkennen.

Was die Ausbildung selbst anlangt, so halte auch ich den Gedanken, die Laienrichter „ohne vorherige Ausbildung" nochmals auf ihre Eignung zu prüfen (S.6), für gut. Bezüglich der ebendort erwähnten Kommissionen scheinen mir noch einige Fragen nicht ausreichend geklärt. Was heißt „Vertreter des öffentlichen Lebens“? Nach welchen Gesichtspunkten sollen die Kommissionen entscheiden? Diese Frage hängt eng mit der Frage zusammen, unter welchen Gesichtspunkten die drei erstgenannten Mitglieder der Kommission vorgeschlagen und ausgewählt (von wem?) werden sollen. Ist das richtig, so würden bei

\footnotetext{
${ }^{13}$ Es folgen Abschnitte zur Finanzierung der auszubildenden Volksrichter und zu den Kosten der Ausbildung.

${ }^{1}$ Dokument 6.
} 
der „Abschlußprüfung “, d.h. einer juristischen Prüfung, nur zwei von fünf Prüfern Juristen sein. Dieses Bedenken wiederholt sich bei der der Kommission zugedachten Entscheidung darüber, ob ein Bewerber ganz oder zum Teil von der Ausbildung freizustellen ist. Wenn sodann vorgeschlagen worden ist, daß jene zwei Mitglieder Angehörige der „allgemeinen Prüfungskommission“ sein sollen, so kann hieraus geschlossen werden, daß die bei der Aufnahme (Auslese) tätigen Vertreter der Justizverwaltungen nicht den „allgemeinen Prüfungskommissionen" angehören. Das würde insgesamt bedeuten, daß im ganzen Verfahren die Prüfungsämter unbeteiligt blieben. Ich würde ein solches Ergebnis sehr bedauern, weil damit die Trennung der beiden Richterkategorien schon in der Wurzel festgelegt und der Tätigkeit der Prüfungsämter ein für sie in jedem Falle lehrreiches Beispiel vorenthalten würde.

Auf Seite 7 ist betont, daß die Volksrichter mit vorheriger Vorbildung jedenfalls so lange notwendig sein werden, als normaler Nachwuchs laufend nicht zur Verfügung steht. Ich nehme an, daß das Gleiche erst recht für Volksrichter ohne vorherige Ausbildung gelten soll.

Zur Seite 8: Ich vermag nicht zu erkennen, inwiefern die neuen Studienpläne eine ausreichende ideologische Vorbereitung der Mitarbeit der juristischen Fakultäten zur Folge haben sollen, zumal mir bisher die neuen Studienpläne nicht bekannt sind. Von größerer, vielleicht ausschlaggebender Bedeutung scheint mir die Frage der Persönlichkeiten. Mancher für die wissenschaftliche Lehre sonst unbedenklich geeigneter Professor besitzt vielleicht nicht die Gabe der Konzentration des Stoffes in dem hier notwendigen Maße, auch nicht das Einfühlungsvermögen, das hier besonders Vorbedingung des Erfolges sein dürfte. Daran ändern aber Studienpläne kaum etwas, ganz abgesehen davon, daß der Bestand der Fakultäten zur Zeit große Lücken aufweist, ohne daß - nach 12 Jahren Mißwirtschaft - ausreichender Ersatz vorhanden ist. Auch wenn diese Besorgnis nicht begründet sein sollte, scheint es mir richtiger, zu hauptsächlichen Trägern der geplanten Ausbildung Praktiker zu machen, die sich in den praktischen Lehrgängen der Fakultäten besonders bewährt haben. Die gesamte Ausbildung könnte auch dann im Rahmen der Fakultäten erfolgen, wenn das für wesentlich gehalten wird.

Was die Frage der Ausbildung betrifft, so dürfen Vorschläge, die 10-Tage-Kurse und Fernbriefunterricht zum Gegenstand haben, in Anbetracht des hohen, mit dem Gesamtplan verbundenen Risikos von vornherein starke Bedenken gegen sich haben. Die Verschiedenheit der "gewissen theoretischen Vorbildung" und "gewissen Vorkenntnisse" (S.9) erhöht offenbar solche Bedenken. Kaufleute haben, auch wenn sie als Diplom-Kaufleute vorgebildet sind, eine ganz andere theoretische Schulung als Lehrer, und jedenfalls letztere haben keine weitergehenden Vorkenntnisse als etwa aus dem bürgerkundlichen Unterricht an Fortbildungs-, Berufs- und Handelsschulen. Die Basis der gesamten Ausbildung würde also wohl so vielgestaltig sein, als Berufe, Anschauungen und Fähigkeiten vorhanden sind. In gewissem Maße könnten diese Schwierigkeiten durch die gleichmäßige Betätigung in der praktischen gerichtlichen Arbeit ausgeglichen werden. Eine Beschäftigung mit „Kanzlei- und Registraturarbeiten sowie Protokoll führen“ wäre freilich in diesem Sinne kein geeignetes Mittel. Auch davon abgesehen, glaube ich nicht, daß drei Monate Tätigkeit eines mittleren Beamten bei einer so gedrängten und inhaltlich weitschichtigen Ausbildung ein geeignetes Ausbildungsmittel sind oder auch nur von den Beteiligten als ein solches empfunden werden würde.

Zur Ausbildung der Volksrichter „ohne vorherige Vorbildung“ möchte ich noch eine Frage stellen: Bringen wir nicht diese Richter in eine innerlich ungemein schwierige 
Lage, wenn wir ihnen richterliche Gewalt schon in dem Augenblicke einräumen, in dem wir ihnen den ersten systematischen Einblick in die ihrer Amtstätigkeit zu Grunde liegenden Wissensgebiete gewähren? Meister und Lehrling zu gleicher Zeit zu sein, bedeutet eine Aufgabe, der nur sehr starke Charaktere nicht von vornherein erliegen dürften.

Ich habe Abschriften dieser Bemerkungen außer an den Herrn Minister den sachlich beteiligten Herren Vizepräsidenten Kleikamp und Direktor Dr.Winkelmann zugehen lassen.

gez. Wende

\section{Dokument $8^{1}$}

Ordnung betreffend die Einrichtung von juristischen Fachschulen, o. D.

BAP, DP1 SE Nr.3561, Durchschrift

I.

Der gegenwärtige Mangel an Richtern und Staatsanwälten, die den Anforderungen für den Aufbau eines neuen deutschen Rechtswesens entsprechen, macht - unbeschadet der auf akademischem Studium beruhenden, mit der Assessorprüfung abschließenden Ausbildung - die Heranziehung von Personen zu diesen Berufen notwendig, die für sie nach Interesse und Begabung geeignet sind, aber keine akademische Bildung erworben haben. In Betracht kommen hierfür alle Deutschen, Männer und Frauen, die das 30. Lebensjahr vollendet und ihre antifaschistisch-demokratische Haltung einwandfrei bewiesen haben.

II.

Die Ausbildung solcher Personen für den Beruf eines Richters oder Staatsanwalts erfolgt in einem Lehrgang von sechsmonatiger Dauer. Für jede Provinz und jedes föderale Land der sowjetischen Besatzungszone in Deutschland wird ein Lehrgang, tunlichst am Sitze des jeweils zuständigen Oberlandesgerichts oder einer Universität, eingerichtet.

III.

Die Lehrgänge werden am 2.Januar 1946 eröffnet.

IV.

Wirtschaftliche Träger der Lehrgänge, insbesondere hinsichtlich der Kosten der Lehrkörper, der Räume und Lehrmittel, sowie der Versorgung und Unterbringung der Teilnehmer, sind die Provinzial- (Landes-)Verwaltungen.

V.

Die Auswahl der Bewerber in der nach dem Bedarf der Provinz bezw. des Landes jeweils erforderlichen Zahl erfolgt durch die zugelassenen politischen Parteien. Diese meiden ihre Vorschläge der betreffenden Provinzial- (Landes-)Verwaltung, die die Kursusteilnehmer einberuft. An einem Kursus sollen nicht mehr als 40 Personen teilnehmen.

${ }^{1}$ Das Dokument wurde von Wende am 22.11. 1945 paraphiert. 
VI.

Den Richtern und Staatsanwälten ohne akademische Vorbildung, die sich bereits im Amt befinden, steht es frei, ob sie zur Ergänzung ihrer juristischen Bildung unter Beurlaubung aus ihrem Amt an einem Lehrgang teilnehmen oder sich neben ihrer beruflichen Tätigkeit selbst fortbilden wollen. Es wird in Aussicht genommen, hierfür sowie für die von ihnen abzulegende Prüfung noch besondere Anordnungen zu treffen.

VII.

Die Bildung der Lehrkörper bleibt den Verwaltungen der Provinzen bezw. Ländern überlassen. Als Lehrer werden außer Richtern und Staatsanwälten, die in erster Linie von den Präsidenten der Oberlandesgerichte vorzuschlagen sein werden, vorzugsweise Praktiker mit besonderen Erfahrungen in der Schulung junger Juristen, aber auch, wo die örtlichen Verhältnisse es gestatten, Universitätslehrer in Frage kommen.

VIII.

Der Lehrgang wird zweckmäßig derart gegliedert, daß in den ersten beiden Monaten Sachgebiete behandelt werden, die gleichmäßig für künftige Richter und Staatsanwälte von Bedeutung sind. In den letzten 4 Monaten erfolgt dann die Ausbildung von Richtern für die Zivilgerichtsbarkeit gesondert von derjenigen der zukünftigen Strafrichter und Staatsanwälte.

In den ersten zwei Monaten ist demnach folgender Lehrplan innezuhalten:

1.) Grundlagen des Staats- und Verwaltungsrechts 4 Wochenstd.

2.) Gesellschaft, Wirtschaft und Recht (konversatorisch) 4 Wochenstd.

3.) Grundbegriffe des Bürgerlichen Rechts und Allgemeiner Teil des BGB (konversatorisch)

4.) Grundbegriffe des Strafrechts 6 Wochenstd.

5.) Grundzüge des Arbeits- und Wirtschaftsrechts (konversatorisch)

4 Wochenstd.

6.) Gerichtsverfassungsgesetz und Justizorganisation

4 Wochenstd.

4 Wochenstd.

Der Ausbildungsplan für die zukünftigen Zivilrichter umfaßt in den letzten 4 Monaten folgende Veranstaltungen:

1.) Recht der Schuldverhältnisse

2.) Sachenrecht

3.) Familien- und Erbrecht

4.) Grundzüge des Handels-, Wechsel- und Genossenschaftsrechts (konversatorisch)

5.) Zivilprozeß einschließlich der Grundlagen der Zwangvollstreckung und des Konkursrechts

6.) Freiwillige Gerichtsbarkeit

Je 5 Wochenstunden unter Einteilung des Stoffs nach dem Ermessen des Kursusleiters

3 Wochenstd.

8 Wochenstd. unter Einteilung des Stoffs nach dem Ermessen des Kursusleiters

Neben dieser Ausbildung in Kursen geht der Besuch von Gerichtssitzungen mit nachfolgender Besprechung einher.

Die Ausbildung der zukünftigen Strafrichter und Staatsanwälte umfaßt folgende Gebiete: 
1.) Strafrecht Allgemeiner Teil

2.) Strafrecht besonderer Teil

3.) Strafrechtliche Nebengesetze

4.) Strafprozeß

5.) Jugendstrafrecht

6.) Verbrechensursache und Verbrechensbekämpfung

7.) Kriminalistik (Untersuchung $u$. Verfolgung von Verbrechen)

8.) Gerichtliche Medizin

9.) Strafvollzug

10.) Grundzüge des Bürgerlichen und Zivilprozeßrechts zu 1.) u. 2.) je 4

zu 3.) 3 Wochenstunden

unter Einteilung des

Stoffs nach dem Ermessen des Kursusleiters

6 Wochenstunden

1 Wochenstunde

je eine Wochenstunde unter Einteilung des

Stoffs nach dem Ermessen

des Kursusleiters

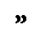

s

4 Wochenstunden

Für den Besuch geeigneter Gerichtssitzungen gilt das gleiche wie für die Zivilrichter.

IX.

Zur Ergänzung des theoretischen Unterrichts werden den Kursusteilnehmern je nach den Fortschritten ihrer Ausbildung schriftliche Aufgaben gestellt werden, die dann im Rahmen der Vorlesungen zu besprechen sind.

$\mathrm{X}$.

Nach Ablauf der ersten drei Monate soll der Leiter des Lehrgangs Teilnehmer, bei denen infolge geringer Begabung und Leistungen nach dem Urteil ihrer Lehrer ein erfolgreicher weiterer Besuch des Lehrgangs ausgeschlossen erscheint, hiervon in gemeinsamer Aussprache mit den beteiligten Lehrern verständigen, gegebenenfalls ihnen den Rücktritt vom Lehrgang empfehlen.

XI.

Über den erfolgreichen Besuch des Lehrgangs weisen sich die Teilnehmer in einer am Schluß des Lehrgangs abzulegenden Prüfung aus. In dem schriftlichen Teil der Prüfung werden unter Aufsicht zwei Arbeiten angefertigt, die für künftige Strafrichter und Staatsanwälte dem Strafrecht, für künftige Zivilrichter dem Zivilrecht, je einschließlich des Straf- bezw. Zivilprozeßverfahrens zu entnehmen sind. In der mündlichen Prüfung ist von jedem Teilnehmer ein Vortrag aus Akten zu halten, die ihm zur Vorbereitung 48 Stunden vor dem Beginn der Prüfung zugestellt werden. Daran schließt sich die weitere mündliche Prüfung in freier Rede und Gegenrede an.

XII.

Wird die Prüfung nicht bestanden, so hat der Prüfungsausschuß darüber zu entscheiden, ob eine Wiederholung der Prüfung nach Teilnahme an einem neuen Lehrgang zugelassen wird.

Der Prüfungsausschuß besteht aus 4 Prüfern einschließlich des Vorsitzenden. Den Vorsitz in der mündlichen Prüfung führt ein Vertreter des Chefs der Deutschen Zentralen Justizverwaltung für das Gebiet der Sowjetischen Besatzungszone in Deutschland. Die Mitglieder des Ausschusses sind der Leiter des Lehrganges und ein weiteres Mitglied des Lehrkörpers, das von diesem durch Beschluß zu bestellen ist, sowie ein Vertreter der beteiligten Landesjustizverwaltung. Die Entscheidungen des Prüfungsausschusses werden mit Stimmenmehrheit getroffen. Bei Stimmengleichheit entscheidet die Stimme des Vorsitzenden. 


\section{Dokument 9}

Schreiben der Landesverwaltung Sachsen/Abteilung Justiz an Chef der DJV; Dresden, den 24.11. 1945

\section{$B A P, D P 1$ SE Nr.3478, Original}

Betrifft: Heranbildung von Laien zu Richtern.

Auf die dortigen Schreiben vom 13. Nov. 1945, Zeichen II. A. R. 45.45., und vom 16. Nov. 1945, Zeichen VI. $63.45^{1}$.

Dem Ressort Justiz der Landesverwaltung Sachsen ist am 29. Oktober 1945 durch den 1.Vizepräsidenten der Landesverwaltung, Fischer ${ }^{2}$, eine Anregung des Chefs der Verwaltung der Sowjetischen Militärischen Administration für das Bundesland Sachsen, Herrn Generalmajor Dubrowski ${ }^{3}$, übermittelt worden, nach der Kurse für die Ausbildung von Richtern eingerichtet und binnen einer Woche ausführliche Lehrpläne, Listen der in Betracht kommenden Dozenten sowie Vorschläge für die technische Planung vorgelegt werden sollten. Die Kurse sollten einen Zeitraum von 7 bis 8 Monaten und eine Hörerschaft von 120 bis 150 Personen umfassen, die ausschließlich aus überzeugten Antifaschisten bestehen und gemeinsam mit den antifaschistischen Parteien ausgewählt werden sollten.

Auf Grund dieser Anregung hat die Landesverwaltung Sachsen - Justiz - am 31. Oktober 1945 die vier im Antifaschistischen Block zusammengeschlossenen politischen Parteien um Namhaftmachung von je etwa 30 Anwärtern - Männer und Frauen - im Alter von 25 bis 35 Jahren gebeten, die die nötige geistige Regsamkeit, Erfahrung im Umgang mit Menschen, Gewandtheit im mündlichen und schriftlichen Ausdruck und die zur Wahrnehmung einer selbständigen Stellung in der Rechtspflege erforderlichen Charaktereigenschaften besäßen, um den Lehrgang mit Erfolg besuchen und später ein Richteramt bekleiden zu können.

Die Landesverwaltung - Justiz - hat ferner nach eingehender Beratung mit führenden Angestellten des öffentlichen Dienstes und Vertretern der Rechtsanwaltschaft einen Lehrplan aufgestellt und ihn mit einem Begleitschreiben vom 6. November 1945 an den Chef der Verwaltung der Sowjetischen Militärischen Administration für das Bundesland Sachsen übermitteln lassen. Abschriften dieses Schreibens und des Lehrplans werden in der Anlage zur näheren Unterrichtung über die geplante Ausgestaltung des Lehrgangs beigefügt ${ }^{4}$. Aus ihnen ergibt sich auch zugleich die Beantwortung der im dortigen Schreiben vom 13 . November $1945^{5}$ gestellten Einzelfragen. Ergänzend wird noch bemerkt, daß die Landesverwaltung - Justiz - die für die Einberufung in Betracht kommenden Teilnehmer

${ }^{1}$ Die DJV hatte das Schreiben vom 13.11. aufgrund der Notiz in der Täglichen Rundschau vom 2.11. 1945 und das Schreiben vom 16.11. aufgrund eines Artikels in Der Morgen vom 16.11. 1945 an die Abteilung Justiz der Landesverwaltung Sachsen gerichtet, BAP, DP1 SE Nr.3478. Zu diesen Vorgängen siehe Einleitung, S. 21.

2 Kurt Fischer (KPD/SED), 1. Vizepräsident der Landeswerwaltung Sachsen.

${ }^{3}$ Dimitri Georgewitsch Dubrowski, stellvertretender Chef für Zivilangelegenheiten der Sowjetischen Militäradministration in Sachsen.

4 Zum Schreiben an Dubrowski vom 6.11. 1945 siehe Einleitung, S.21 f. Der Lehrplan war im Bestand DP1 des Bundesarchivs, Abteilungen Potsdam, nicht auffindbar.

${ }^{5}$ Siehe Anm.1. 
aus der Gesamtzahl der Bewerber nach einer persönlichen Vorstellung auszuwählen beabsichtigt, die unter maßgeblicher Mitwirkung des mit der wissenschaftlichen Leitung und Ausgestaltung des Lehrgangs beauftragten Herrn Generalstaatsanwalts Dr. Schroeder ${ }^{48}$ stattfinden soll.

Dr. Uhle

Vizepräsident ${ }^{49}$.

\section{Dokument 10}

Schreiben der Rechtsabteilung der SMAD an Chef der DJV; Berlin, den 17.12. 1945

BAP, DP1 SE Nr.3478, Übersetzung

Von einzelnen Organen der örtlichen Selbstverwaltung ist die Frage wegen Ausbildung von Richtern und Staatsanwälten für die deutschen Gerichte durch organisieren [sic] von juristischen Kursen angeregt worden ${ }^{1}$.

Der oberste Chef der SMA in Deutschland hat diese Maßnahme für gut befunden und folgende Anweisung gegeben:

1. Es sind beständige juristische Kurse mit einem Kontingent von je 30 bis 40 Lernenden und einem Lehrgang von 6 Monaten zu organisieren.

2. Die Kurse sind aus Personen beiderlei Geschlechts aus der Zahl aktiver Antifaschisten, die mindestens die Volksschulbildung besitzen und über 25 Jahre alt sind, zu komplettieren.

3. Die Verantwortung für die Komplettierung der Kurse wird den Präsidenten der Provinzen und föderalen Länder übertragen. Die Präsidenten der Oberlandesgerichte sind zu den Komplettierungsmaßnahmen hinzuzuziehen.

4. Die Kurse sind auf Kosten der Budgets der Selbstverwaltungen der Provinzen und föderalen Länder zu unterhalten, sowie mit dem vollen Bestande der Lehrkräfte und Unterrichtsutensilien auszurüsten. Den Hörern sind die erforderlichen Bedingungen zu gewährleisten.

5. Die Leiter der juristischen Kurse sind von den Präsidenten der Provinzen (föderalen Länder) auf Vorschlag der Präsidenten der Oberlandesgerichte zu ernennen.

6. Personen, die die Kurse beendet haben, sind, nach Ablegung der Prüfung, der Arbeit bei den Gerichten und den Staatsanwaltschaften zu überweisen.

7. Die Kurse sollen ab 1.2. 1946 mit der Arbeit beginnen².

48 John Ulrich Schroeder (SPD/SED), Generalstaatsanwalt von Sachsen.

49 Reinhard Uhle (LDP), Vizepräsident der Landesverwaltung Sachsen, zuständig für Justiz und Gesundheitswesen.

1 Auf welche Anfragen hier angespielt wird, ist unklar: siehe dazu Einleitung, S. 23.

2 Pfannkuch, Volksrichterausbildung, hat Punkte 1-7 als Anlage Nr. 5, S. 147, ohne Datumsangabe abgedruckt. Es handelt sich dabei freilich nicht, wie sie schreibt, um einen "Befehl“ der SMAD, sondern, wie es in dem Dokument heißt, um eine "Anweisung". 
Ihnen wird die methodische Leitung dieser Kurse sowie die Aufstellung der Programme und Lehrpläne übertragen.

Die Programme und Lehrpläne bitte ich mir zum 1. Januar 1946 zur Bestätigung vorzulegen.

Der Chef der Rechtsabteilung der SMA in Deutschland J. Karasjew

\section{Dokument 11}

Schreiben Kleikamp an Wende; Berlin, den 21.1. $1946^{1}$

BAP, DP1 SE Nr.3561, Durchscbrift

Betrifft: Lehrgänge für Laienrichter.

Die in Ihrem Vermerk vom 15. d.Mts. - VI.28.46 - festgehaltene Mitteilung des Herrn Majors Nikolajew von der Rechtsabteilung der SMA in Karlshorst ${ }^{2}$ ist am gleichen Tage, dem 12. d.Mts. auch mir gemacht worden. Herr v. Stackelberg, der an dieser Unterhaltung mit Herrn Major Nikolajew als Dolmetscher teilgenommen hat, hat in einer auf meine Bitte von ihm nach der Erinnerung angefertigten Niederschrift hierüber folgendes vermerkt:

„Wegen der Kurse sei jedoch besonders zu beachten, daß sie nur als temporäre, zeitbedingte Maßnahme zur Deckung des augenblicklichen tatsächlichen Bedarfs an Richtern und Staatsanwälten gedacht seien. Im Prinzip vertrete die Rechtsabteilung den Standpunkt, daß die Richterposten mit vollwertigen Richtern mit juristischer Hochschulbildung zu besetzen seien. Die Rechtsabteilung wünsche und beabsichtige, dieses Ziel auch in Zukunft zu verfolgen und hoffe, daß schon nach einigen Jahren die Universitäten die erforderliche Anzahl von Kandidaten hierfür liefern würden. Dieses sei besonders bei der Komplettierung der Kurse im Auge zu behalten. Die Zahl der Teilnehmer müßte daher entsprechend dem augenblicklichen tatsächlichen Bedarf an neuen Richtern und Staatsanwälten festgesetzt werden. ${ }^{\text {3 }}$

Diese Festlegung deckt sich mit meiner Erinnerung.

Andererseits darf nicht außer Acht gelassen werden, daß es sich hier nur um eine mündliche Mitteilung handelt, die auch nicht der Gegenstand einer selbständigen Eröffnung war, sondern um eine Meinungsäußerung aus Anlaß der Erwähnung der großen Teilnehmerzahl, mit der der Lehrgang im Lande Sachsen eröffnet werden soll. Ich würde es daher für untunlich halten, wenn wir - unbeschadet eines Hinweises an das Land Sachsen,

\footnotetext{
${ }^{1}$ Kleikamp sandte das Schreiben an Wende "durch die Hand des Amtschefs".

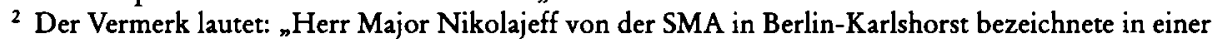
Besprechung mit dem Herrn Amtschef vom 12. d.Mts. die Lehrgänge zur Ausbildung von Richtern und Staatsanwälten als nichtständige ,temporäre' Einrichtungen zur Deckung des gegenwärtigen Bedarfs und bemerkte dabei, daß die bisherige (akademische) Vorbildung an sich erhalten bleiben sollte." BAP, DP1 SE Nr.3478.

${ }^{3}$ Der gesamte Vermerk vom 14.1. 1946 in: BAP, DP1 SE Nr.3561.
} 
dessen Formulierung ich mir vorbehalte ${ }^{4}$ - nach außen von dieser Eröffnung des Herrn Major Nikolajew Gebrauch machen würden.

Die noch nicht aufgehobene schriftliche Anweisung, die die Grundlage für diese ganze Einrichtung bildet, lautet nach wie vor unverändert dahin, daß es sich bei diesen Kursen um eine beständige ${ }^{5}$ Einrichtung handeln soll. Jedenfalls bitte ich, alle schriftlichen amtlichen Äußerungen, die über diese Frage aus unserem Hause herausgehen, gleichgültig aus welchem Anlaß, dem Herrn Amtschef oder mir zur Zeichnung vorzubehalten und diesem Gesichtspunkt auch bei etwaigen mündlichen Erklärungen Rechnung zu tragen ${ }^{6}$.

\section{Dokument 12}

Schreiben des Präsidenten der Provinzialverwaltung Mark Brandenburg an DJV; Potsdam, den 26.3. 1946

$B A P, D P 1 S E$ Nr.3478, Original

Betrifft: Lehrgang zur abgekürzten Ausbildung von Richtern und Staatsanwälten Dortiges Schreiben vom 8.3. 1946 - VI 112/46 -

Das Schreiben vom 8. März ${ }^{1}$ erklärt das grundlegende Lehrprogramm vom 28.12.45 - VI $106 / 45$ - $^{2}$ für allgemein verbindlich. Danach wäre auch die in dem ursprünglichen Lehrprogramm nur als „zweckmäßig“ empfohlene Teilung des Lehrganges in einen Zweig für zukünftige Strafrichter und Staatsanwälte und einen Zweig für Richter der zivilen Gerichtsbarkeit unbedingt durchzuführen. Diese Weisung der SMA in Karlshorst kommt überraschend, nachdem bereits in einer Besprechung der mit der Einrichtung der Lehrgänge beauftragten Oberlandesgerichtspräsidenten am 12.1. 1946 von allen dabei vertretenen Provinzen und Ländern übereinstimmend gegen diese Teilung Stellung genommen wurde, ohne daß Herr Ministerialdirektor Dr. Wende als Leiter der Beprechung dies damals zum Anlaß genommen hätte, die Teilung des Lehrganges als Befehl der SMA zu kennzeichnen. Die anwesenden Vertreter der Provinzen und Länder gaben damals eindeutig und unwidersprochen ihrer Auffassung Ausdruck, daß die Teilung des Lehrganges von der Deutschen Zentralen Justizverwaltung nur unverbindlich empfohlen sei, und Herr Dr. Wende unterstrich dies, indem er erklärte, daß die Durchführung der Lehrgänge grundsätzlich Sache der Länder und Provinzen sei, und daß diese selbst ihre Erfahrungen

${ }^{4}$ Siehe Kleikamp an Wende, 14.2. 1946, BAP, DP1 SE Nr.3478.

${ }^{5}$ Kleikamp leitete dies vermutlich aus Punkt 1 der Anweisung vom 17.12. 1945 (Dokument 10) ab, in der es hieß, daß „beständige juristische Kurse" einzurichten seien.

${ }^{6}$ Auf dem Original des Vermerks, BAP, DP1 SE Nr.3478, befindet sich folgender handschriftlicher Vermerk Schiffers vom 23.1. 1946: „Ich bin mit dieser taktischen Zurückhaltung gegenüber nichtrussischen Stellen durchaus einverstanden, bemerke aber zur Sache selbst, daß gleich zu Beginn der Verhandlungen über diesen Gegenstand G. Mainalow [?] nachdrücklich erklärte, die akademische Ausbildung müsse die Regel bilden und Ausnahmen seien nur in ganz engem Rahmen, in einer Anmerkung" vorgesehen." Vgl. dazu auch Einleitung, S. 24 f.

1 Das Schreiben vom 8.3. 1946 liegt nicht vor; es enthielt offensichtlich die Mitteilung, daß der am 24.1. 1946 von der SMAD bestätigte Lehrplan (BAP, DP1 VA Nr. 1069, Bl.11) verbindlich sei.

${ }^{2}$ Siehe Chef der DJV an Landes- und Provinzialverwaltungen/Abt. Justiz, 28. 12. 1945, BAP, DP.1 SE Nr.3561. 
sammeln müßten. Auf Grund der Besprechung am 12.1. hat die Justizabteilung der Provinzialverwaltung Mark Brandenburg einen Lehrplan ausgearbeitet, der keine Teilung des Lehrganges vorsieht, und der Deutschen Zentralen Justizverwaltung zur Kenntnisnahme vorgelegt. Auch hierauf erfolgte kein Widerspruch. Die Vorlesungen haben dann am 18. 2. 1946 nach dem Lehrplan der Provinzialverwaltung begonnen.

Bei dieser Sachlage kann ich wohl von der Annahme ausgehen, daß die Deutsche Zentrale Justizverwaltung auf einer Durchführung der ursprünglich empfohlenen Zweiteilung des Lehrganges nicht bestehen wollte, sich aber nunmehr durch das unvermutete Eingreifen der SMA hierzu genötigt sieht. Um eine wirklich erfolgreiche Durchführung des Lehrganges zu erreichen, halte ich es für geboten, die Frage der Teilung des Lehrganges nochmals mit der Rechtsabteilung der SMA in Karlshorst zu erörtern.

Nach meiner Auffassung sprechen gegen eine Teilung des Lehrganges in einen strafrechtlichen und einen zivilrechtlichen $Z$ weig folgende Gründe:

1) Vom Standpunkt der Justizverwaltung:

Die künftigen Richter und Staatsanwälte werden nach der verhältnismäßig kurzen Ausbildung von 6 Monaten zunächst noch einer Anleitung und Führung durch erfahrene Berufskollegen bedürfen und sollen deshalb längere Zeit an größeren Gerichten als Beisitzer im Kollegialgericht oder als Staatsanwälte mit beschränktem Zeichnungsrecht Verwendung finden. Es scheint mir aber nicht tragbar, den Einsatz der neuen Richter und Staatsanwälte für immer auf die wenigen größeren Gerichte der Provinz zu beschränken, weil mit Nachwuchs aus dem normalen Ausbildungsgang auf lange Sicht nicht zu rechnen ist, die gegenwärtig eingesetzten stark überalterten Kräfte aber zwangsläufig nach und nach ausfallen. Es muß daher davon ausgegangen werden, daß früher oder später wenigstens die Begabtesten unter diesen Nachwuchsjuristen auch als Richter bei kleinen Amtsgerichten eingesetzt werden müssen. Schafft man aber durch die Art der Ausbildung einen neuen Typ von vorwiegend strafrechtlich oder zivilrechtlich vorgebildeten Juristen, so scheiden diese als Richter bei den kleineren Amtsgerichten - und das sind in der Mark Brandenburg weitaus die meisten - von vornherein aus, weil dort jeder Richter sowohl im Strafrecht als auch im Zivilrecht tätig werden muß. Nur bei einer einheitlichen Grundausbildung der Lehrgangsteilnehmer wird $\mathrm{m}$. E. der neben der politischen Zielsetzung wichtigste Zweck des Lehrganges voll erreicht werden, nämlich eine fühlbare Erleichterung in der Besetzung der Planstellen für Richter und Staatsanwälte.

2) Vom pädagogischen Standpunkt:

Die scharfe Trennung des Strafrechts vom Zivilrecht ist größtenteils theoretischer Natur. Tatsächlich muß der Strafrichter und Staatsanwalt immer wieder zivilrechtliche Vorfragen entscheiden und umgekehrt der Zivilrichter Normen des Strafrechts anwenden. Wie soll der Richter und Staatsanwalt die alltäglichen Vermögensdelikte, wie Unterschlagung und Betrug richtig beurteilen, wie soll er vollends einen Untreuefall zutreffend entscheiden können, ohne einigermaßen fundierte Kenntnisse im Zivilrecht zu besitzen? Da dieser Ausbildungslehrgang im Gegensatz zur Universitätsausbildung besonders auf die praktische Tätigkeit zugeschnitten ist, sollte an dem praktischen Erfordernis, das Zivil- und Strafrecht in seinem organischen Zusammenhang zu erkennen und anzuwenden, im Unterricht nicht vorübergegangen werden. Bei einer Teilung des Lehrganges müßte aber der Teilnehmer je nach seinem Ausbildungsziel im Zivilrecht oder Strafrecht mit notgedrungen kärglichen Grundlagen auskommen, die ihn m. E. nicht befähigen, die auftauchenden Fragen aus dem „Nebengebiet“ zu lösen. 
3) Vom psychologischen Standpunkt:

Die in der Verfügung vom 28.12. 1945 enthaltene Ankündigung über die „beabsichtigte“ Gliederung des Lehrganges enthält keinen Hinweis, ob der einzelne Teilnehmer sich selbst für den strafrechtlichen oder zivilrechtlichen Zweig entscheiden, oder ob die Provinzialverwaltung hier nach ihrem Ermessen und Bedürfnis die Teilung vornehmen soll. Man wird aber die Lehrgangsteilnehmer auf jeden Fall vor dieser Entscheidung hören müssen. Diese werden sich jedoch mit wenigen Ausnahmen nach den vorgesehenen 2 Monaten noch nicht darüber klar sein, in welche Richtung sie Interesse und Begabung treibt. Die Teilung des Lehrganges ist jetzt unter den Teilnehmern zur Diskussion gestellt worden. Bis auf einen lebnten alle den Gedanken einer Teilung entschieden ab, schon weil sie die Tragweite ihrer Wahl nicht übersehen können. Sie empfinden den Zwang, sich zu spezialisieren, ohne einen endgültigen Überblick über das gesamte Recht gewonnen zu haben, als drückend. Das ist eine wenig günstige Voraussetzung für ihren Lerneifer und ihre Aufmerksamkeit.

Vor allem aber muß nach den bisherigen Erfahrungen mit bereits eingesetzten Volksrichtern und nach zahlreichen Äußerungen aus dem Kreise der gegenwärtigen Lehrgangsteilnehmer damit gerechnet werden, daß gerade diese neuen Juristen peinlich darauf bedacht sind, sich keine Blöße zu geben, und nichts mehr befürchten, als nicht für voll genommen zu werden. Die Erkenntnis, nur auf einem Teilgebiet ausgebildet zu sein, wird in den meisten von ihnen ein Minderwertigkeitsgefühl entstehen lassen, das ihre Eignung als Richter oder Staatsanwalt von vornherein in Frage stellt. Denn ein Richter oder Staatsanwalt, der sich seiner Aufgabe nicht voll gewachsen fühlt, wird unsicher auftreten, eine Erscheinung, die gerade bei dem im Brennpunkt auch des politischen Interesses stehenden Volksrichter und Volksstaatsanwalt unbedingt vermieden werden muß. Ich bezweifle, daß es pädagogisch möglich sein wird, dem Lehrgangsteilnehmer dieses Minderwertigkeitsgefühl mit Erfolg auszureden. Der auf allen Gebieten des Rechts ausgebildete Jurist ist auch als Berufsvorstellung viel zu fest im Volksbewußtsein verankert, als daß es möglich erschiene, die Lehrgangsteilnehmer mit der Hoffnung auf eine spätere Aussicht, die fehlenden Kenntnisse nachzuholen, zu vertrösten.

4) Vom politischen Standpunkt:

Die Ausbildung von Werktätigen aus dem Volke ohne einschlägige Vorkenntnisse zu Richtern und Staatsanwälten ist eine aktuelle politische Forderung. Ihre Erfüllung führt nur dann zu einem gesunden, für die Gesamtheit wertvollen Ergebnis, wenn die neuen Volksrichter und Volksstaatsanwälte in diesem Volk selbst mindestens die gleiche Achtung und Autorität genießen wie der Volljurist bisheriger Prägung in diesen Ämtern. Eine völlige äußere Gleichstellung des Nachwuchses aus dem Ausbildungslehrgang ist deshalb geplant. Diese Maßnahme wird aber wirkungslos bleiben, wenn schon die Ausbildungsweise der Volksrichter und Volksstaatsanwälte sie als schwächere Fachkräfte charakterisiert. Zwingt man den künftigen Richtern und Staatsanwälten eine vorzeitige Spezialisierung auf, so wird dies nicht verborgen bleiben, sondern die Bevölkerung wird mehr denn je den „halbgebildeten“ Juristen dem Volljuristen gegenüberstellen. Dieses Ergebnis halte ich für politisch untragbar.

Die Gründe, welche für eine vorzeitige Spezialisierung der Ausbildung vorgebracht werden, vermag ich nicht anzuerkennen. Eine gleichmäßige Ausbildung auf allen Rechtsgebieten stellt an die Lehrgangsteilnehmer keine wesentlich höheren Anforderungen als die Spezialisierung, wenn man keine übertriebenen Anforderungen stellt. Bei der geplan- 
ten Art des Einsatzes und der späteren Fortbildung werden die in 6 Monaten fleißiger Arbeit auf allen wichtigen Gebieten zu erwerbenden Kenntnisse bestimmt genügen. Die gegenwärtig ausgewählten Lehrgangsteilnehmer werden nach den bisher gewonnenen Erfahrungen und Erkenntnissen in der Mehrzahl wahrscheinlich in der Lage sein, dieses Ausbildungsziel zu erreichen. Die Kenntnisse eines Volljuristen können sich die Lehrgangsteilnehmer bei allem Fleiß in der kurzen Zeit von 6 Monaten ohnehin keinesfalls erwerben, selbst wenn ihnen die Gelegenheit gegeben wird, sich auf ein Teilgebiet zu beschränken. Deshalb ist eine spätere Fortsetzung der Ausbildung durch Fernkurse, Kurzlehrgänge oder ähnliche Mittel doch auf jeden Fall unbedingt erforderlich. Sind die Lehrgangsteilnehmer einseitig ausgebildet, so würde eine Nachholung des fehlenden Ausbildungszweiges aber auf besondere Schwierigkeiten stoßen, weil dies eine Zusammenfassung in einem neuen, länger dauernden Lehrgang erforderlich machen würde, die neuen Richter und Staatsanwälte also in ihren Stellen durch andere juristische Fachkräfte auf längere Zeit ersetzt oder vertreten werden müßten. Bei der gegenwärtigen Personallage ist dies eine unlösbare Aufgabe. Die Fortsetzung der Ausbildung des heute dem Lehrgang angehörenden Nachwuchses wird m.E. im wesentlichen nur eine Vertiefung bereits bekannten Stoffes, der gebotenen zeitlichen Beschränkung halber jedoch nur in geringem Umfange grundsätzlich Neues bringen können. Ich halte es daher für besser, in jedem Falle die allseitige Ausbildung zu versuchen. Sollte sich wirklich wider Erwarten herausstellen, daß ein gutes Ergebnis des Lehrganges in der gesetzten Frist von 6 Monaten nicht zu erreichen wäre, so wäre es $\mathrm{m}$. E. trotz des dringenden Personalbedarfs immer noch besser, einen Monat zuzugeben, als den Ausweg der verfrühten Spezialisierung zu wählen.

Ich bitte, diese Stellungnahme zu der geplanten Teilung des Lehrganges der Rechtsabteilung der SMA in Karlshorst zur Kenntnis zu bringen und, falls eine schriftliche Erledigung in meinem Sinne nicht möglich erscheint, eine Besprechung mit den Herren der SMA zu vermitteln ${ }^{3}$.

Steinhoff

${ }^{3}$ Der Brief Steinhoffs wurde am 18.4. 1946 an die SMAD-Rechtsabteilung mit der Bemerkung weitergeleitet, daß auf Anweisung der Sowjetischen Militäradministration in Brandenburg der dortige Richterlehrgang ungeteilt ablaufen würde: BAP, DP1 SE Nr.3478.

4 Carl Steinhoff (SPD/SED), Präsident der Provinzialverwaltung Brandenburg, verantwortlich für Justiz und Gesundheit. 


\section{Dokument $13^{1}$}

Schreiben Ulbricht an Chef der Justizabteilung der SMAD, Generaloberst Gen. Kurasow $[\mathrm{sic}]^{2}, 9.3 .1946$

\section{SAPMO, ZPA, NL 182/1185, Bl.40-41, Konzept}

Betrifft: Schulung von Richtern

Wie uns aus der Deutschen Justizverwaltung mitgeteilt wurde, ist seitens Ihrer Abteilung die Durchführung der Schulung zur beschleunigten Heranbildung von Richtern und Staatsanwälten auf der Basis der getrennten Ausbildung von Richtern für Zivilsachen auf der einen Seite und Richtern für Strafsachen auf der anderen Seite vorgeschlagen worden, was zur Folge hat, daß den Absolventen dieser Schulung nur das Recht zusteht, entweder in Zivilsachen oder in Strafsachen amtieren zu können.

Wir erlauben uns, gegenüber dieser Zweiteilung der Ausbildung unsere Bedenken vorzubringen, die auf folgenden Erwägungen beruhen:

1. Die Trennung der Ausbildung in Richter für Zivilsachen und Richter für Strafsachen führt insofern zu einer Abschwächung des Instituts der beschleunigten Heranbildung, als die Absolventen dieser Schulung nicht die volle Richterqualität erhalten, $d$. h. daß sie nicht - wie die alten Berufsrichter - zu allen richterlichen Geschäften herangezogen werden können; was die Position dieser jungen Kader gegenüber den alten Richtern erheblich schwächt und sie nicht zu mit den alten Berufsrichtern gleichberechtigten Richtern werden läßt.

2. Die sachliche Teilung der richterlichen Geschäfte in Zivilsachen und Strafsachen ist nicht bei allen Gerichten durchgeführt. Die unteren Gerichte verfügen vielfach nur über einen Richter, der sowohl die Zivilsachen als auch die Strafsachen zu entscheiden hat. Ein Richter, der für die Entscheidung nur der einen oder der anderen Kategorie kompetent ist, wäre also bei diesen Gerichten gar nicht zu verwenden. Hinzu kommt, daß im Geschäftsverkehr der großen Gerichte mit spezialisierten Kammern (Kammern für Zivilsachen und für Strafsachen) die Richter aushilfsweise oder vertretungsweise oft von der einen in die andere Kammer hinübergezogen werden.

Wir dürfen dabei auf folgendes hinweisen:

Unabhängig von dem seitens der Zentralen Justizverwaltung ausgearbeiteten Plan, der eine Zweiteilung der Ausbildung in Zivilsachen und Strafsachen vorsah, haben die Landes- bezw. Provinzial-Justizverwaltungen, die die Organisierung der Schulung in Angriff genommen haben, sich auf den Standpunkt der vollen Ausbildung für beide Rechtsgebiete gestellt und entsprechende Pläne der Schulung zugrunde gelegt. So die Provinz Sachsen und die Provinz Brandenburg. Der Lehrplan des Landes Sachsen sieht nach anfänglicher gemeinsamer Schulung eine Spezialisierung im letzten Stadium der Ausbildung vor.

Sollten Bedenken in der Richtung bestehen, daß das Material beider Rechtsgebiete nicht in den festgesetzten 6 Monaten bewältigt werden kann, so glauben wir, eine Verlängerung der Schulung um 2 bis 3 Monate in Anregung bringen zu können.

Ulbricht

\footnotetext{
${ }^{1}$ Dieses Schreiben ist fast identisch mit einem Entwurf von Karl Polak, dem Leiter der Abteilung Justiz beim SED-Zentralsekretariat, SAPMO, ZPA, IV 2/13/445.

${ }^{2}$ Ulbricht verwechselte offensichtlich Generaloberst Wladimir Kurassow, von Juni bis Oktober 1945 Chef des Stabes der SMAD, mit Karassjow, dem Leiter der SMAD-Rechtsabteilung.
} 